\title{
Triviality of hierarchical $O(N)$ spin model in four dimensions with large $N$
}

\author{
Hiroshi Watanabe \\ Department of Mathematics, Nippon Medical School, \\ 2-297-2, Kosugi, Nakahara, Kawasaki 211-0063, Japan \\ e-mail address: watmath@nms.ac.jp
}

\begin{abstract}
The renormalization group transformation for the hierarchical $O(N)$ spin model in four dimensions is studied by means of characteristic functions of single-site measures, and convergence of the critical trajectory to the Gaussian fixed point is shown for a sufficiently large $N$. In the strong coupling regime, the trajectory is controlled by the help of the exactly solved $O(\infty)$ trajectory, while, in the weak coupling regime, convergence to the Gaussian fixed point is shown by power decay of the effective coupling constant.
\end{abstract}

\section{Introduction}

In order to study a critical spin system with a large coupling constant, it is necessary to control the renormalization group trajectory in a strong coupling regime. In the case of the hierarchical Ising model in four dimensions, the method using characteristic functions of single-site measures was developed and the critical trajectory was shown to converge to a Gaussian measure [7]. This means that the hierarchical Ising model in four dimensions is trivial, namely, the continuum limit of the system is Gaussian.

In the present paper, we study the hierarchical $O(N)$ spin model in four dimensions and show the triviality of this model for a sufficiently large $N$.

Let $N>1$ and $\Lambda>0$ be integers. We consider Dyson's hierarchical spin model 3 with $O(N)$ symmetry on the lattice $\mathcal{L}_{\Lambda}=\{0,1\}^{\Lambda}$ :

$$
\begin{aligned}
\phi_{\theta} & =\phi_{\theta_{\Lambda}, \ldots, \theta_{1}} \in \mathbb{R}^{N}, \quad \theta=\left(\theta_{\Lambda}, \ldots, \theta_{1}\right) \in \mathcal{L}_{\Lambda}, \\
H_{\Lambda}(\phi) & =-\frac{1}{2} \sum_{n=1}^{\Lambda} \frac{1}{(2 \omega)^{n}} \sum_{\theta_{\Lambda}, \ldots, \theta_{n+1}=0,1}\left|\sum_{\theta_{n}, \ldots, \theta_{1}=0,1} \phi_{\theta_{\Lambda}, \ldots, \theta_{1}}\right|^{2}, \\
\langle F\rangle_{\Lambda, h_{0}^{(N)}} & =\frac{1}{Z_{\Lambda, h_{0}^{(N)}}} \int d \phi F(\phi) \exp \left(-\beta H_{\Lambda}(\phi)\right) \prod_{\theta \in \mathcal{L}_{\Lambda}} h_{0}^{(N)}\left(\phi_{\theta}\right), \\
Z_{\Lambda, h_{0}^{(N)}} & =\int d \phi \exp \left(-\beta H_{\Lambda}(\phi)\right) \prod_{\theta \in \mathcal{L}_{\Lambda}} h_{0}^{(N)}\left(\phi_{\theta}\right),
\end{aligned}
$$

where $\beta>0$ and

$$
\omega=2^{2 / d}, \quad d>2 .
$$

For the normalized single site measure density $h_{0}^{(N)}$, we choose

$$
h_{0}^{(N)}(\boldsymbol{x})=\text { const. } \delta(|\boldsymbol{x}|-\sqrt{N} \alpha), \quad \boldsymbol{x} \in \mathbb{R}^{N},
$$

for $\alpha>0$. This spin system is called the $d$ dimensional hierarchical $O(N)$ spin model (slightly different from the version considered in [5] 6]). In what follows, we shall fix the so far arbitrary normalization of the spin variables by

$$
\beta=\frac{\omega-1}{2} .
$$


Hierarchical models are so designed that the block-spin renormalization group transformation $\mathcal{R}$ has a simple form. Define the block spins $\phi^{\prime}$ by

$$
\phi_{\tau}^{\prime}=\frac{1}{\sqrt{2 \omega}} \sum_{\theta_{1}=0,1} \phi_{\tau \theta_{1}}, \tau=\left(\tau_{\Lambda-1}, \ldots, \tau_{1}\right) .
$$

If a function $F(\phi)$ depends on $\phi$ through $\phi^{\prime}$ only, namely, if there is a function $F^{\prime}\left(\phi^{\prime}\right)$ of the block spins such that

$$
F(\phi)=F^{\prime}\left(\phi^{\prime}\right)
$$

then it holds that

$$
\langle F\rangle_{\Lambda, h_{0}^{(N)}}=\left\langle F^{\prime}\right\rangle_{\Lambda-1, \mathcal{R} h_{0}^{(N)}}
$$

where $\mathcal{R}$ is the mapping defined by

$$
\mathcal{R} h(\boldsymbol{x})=\text { const. } \exp \left(\frac{\beta}{2}|\boldsymbol{x}|^{2}\right) \int_{\mathbb{R}^{N}} h\left(\sqrt{\frac{\omega}{2}} \boldsymbol{x}+\boldsymbol{y}\right) h\left(\sqrt{\frac{\omega}{2}} \boldsymbol{x}-\boldsymbol{y}\right) d \boldsymbol{y}, \boldsymbol{x} \in \mathbb{R}^{N} .
$$

Macroscopic properties for the spin system defined by (1.1)-(1.4) are derived from the asymptotic behavior of the renormalization group trajectory

$$
h_{n}^{(N)}=\mathcal{R}^{n} h_{0}^{(N)}, \quad n \geq 0 .
$$

Note that

$$
h_{G}(\boldsymbol{x})=\text { const. } \exp \left(-\frac{1}{4}|\boldsymbol{x}|^{2}\right)
$$

is a fixed point of $\mathcal{R}$, and the expectation $\langle\cdot\rangle_{\Lambda, h_{G}}$ defines a Gaussian measure. We refer to $h_{G}$ as the trivial fixed point of $\mathcal{R}$.

In a weak coupling regime, i.e. in a vicinity of the trivial fixed point $h_{G}$, rigorous methods were developed to control the renormalization group trajectory for $\mathcal{R}$ [5, 6] 11. However, in order to show existence of the critical trajectory (1.9), we need to study the mapping $\mathcal{R}$ in the strong (as well as weak) coupling regime, since the starting point (1.6) is regarded as the strong coupling limit of multi-component $\lambda \phi^{4}$ measure:

$$
\delta(|\boldsymbol{x}|-\gamma)=\lim _{\lambda \rightarrow \infty} \text { const. } \exp \left(-\lambda|\boldsymbol{x}|^{4}+2 \lambda \gamma^{2}|\boldsymbol{x}|^{2}\right) .
$$

In the present paper, we study the trajectory (1.9) and show that the critical trajectory converges to the trivial fixed point $h_{G}$. To be precise:

Theorem 1.1 Let $d=4$. For a sufficiently large $N$, there exists a positive constant $\alpha_{N}$ such that if $h_{n}^{(N)}, n \geq 0$, are defined by (1.6) and (1.9) with $\alpha=\alpha_{N}$, then the sequence of measures $h_{n}^{(N)}(\boldsymbol{x}) d \boldsymbol{x}, n \geq 0$, weakly converges to the trivial fixed point measure $h_{G}(\boldsymbol{x}) d \boldsymbol{x}$ as $n \rightarrow \infty$.

As a result of the above theorem, we see that 'the continuum limit' constructed by using the critical trajectory is Gaussian.

Our proof of Theorem 1.1] is based on the method using characteristic functions of single-site measures developed in [7]. In the present paper, we analyze the $O(N)$ trajectories in the strong coupling regime by explicitly solving $O(\infty)$ trajectories and by estimating differences between $O(\infty)$ and $O(N)$ trajectories. Thus, if $N$ is sufficiently large, we can deal with the renormalization group transformation by hand in contrast with the case $N=1$ solved in [7, in which the analysis in the strong coupling regime is partially computer aided. On the other hand, our argument in the weak coupling regime is essentially the same as 7.

As is stated in Theorem 1.1 we concentrate on the case $d=4$ and put $\omega=\sqrt{2}$, though a parallel argument is possible for $d>4$. 


\section{Outline of the proof}

The proof of Theorem 1.1 is decomposed into three parts:

(1) $O(N)$ trajectory in the weak coupling regime

We obtain a criterion for the trajectory (1.9) to converge to $h_{G}$ assuming that the trajectory has entered a vicinity of $h_{G}$ (Proposition 2.1). Our criterion is stated in terms of characteristic functions and differs from the one given in [11, which is so complicated that it is not clear whether the trajectory starting at (1.6) meets it.

(2) $O(\infty)$ trajectory

We explicitly calculate the $O(\infty)$ trajectories, i.e. the trajectories corresponding to $N=\infty$, and derive the asymptotic behavior of trajectories near the critical point (Proposition 2.2).

\section{(3) From $O(N)$ trajectory to $O(\infty)$ trajectory}

We show that an $O(N)$ trajectory converges to an $O(\infty)$ trajectory as $N \rightarrow \infty$ (Proposition 2.3). Consequently, we can find the critical $O(N)$ trajectory in the vicinity of the critical $O(\infty)$ trajectory for a sufficiently large $N$.

In this section, we describe the outline of our argument and prove Theorem 1.1 assuming Proposition 2.1 Proposition 2.2 and Proposition 2.3 stated below. These propositions are proved in the subsequent sections.

\subsection{Characteristic functions}

We consider characteristic functions of effective measures

$$
\hat{h}_{n}^{(N)}(\boldsymbol{\xi})=\mathcal{F} h_{n}^{(N)}(\boldsymbol{\xi})=\int_{\mathbb{R}^{N}} e^{\sqrt{-1}(\boldsymbol{\xi}, \boldsymbol{x})} h_{n}^{(N)}(\boldsymbol{x}) d \boldsymbol{x}, \quad n=0,1,2, \cdots,
$$

and write the renormalization group transformation for $\hat{h}_{n}^{(N)}$ as

$$
\hat{h}_{n}^{(N)}=\mathcal{F} \mathcal{R F}^{-1} \hat{h}_{n-1}^{(N)}=\mathcal{T} \mathcal{S} \hat{h}_{n-1}^{(N)},
$$

where

$$
\begin{aligned}
\mathcal{S} g(\boldsymbol{\xi}) & =g\left(\frac{1}{\sqrt{2 \omega}} \boldsymbol{\xi}\right)^{2}, \\
\mathcal{T} g(\boldsymbol{\xi}) & =\text { const. } \exp \left(-\frac{\beta}{2} \triangle\right) g(\boldsymbol{\xi}) .
\end{aligned}
$$

In the above, $\triangle$ denotes the $N$ dimensional Laplacian and the constant is chosen so that

$$
\mathcal{T} g(\mathbf{0})=1
$$

holds. Since $\hat{h}_{n}^{(N)}$ has spherical symmetry, we shall often write

$$
\hat{h}_{n}^{(N)}(\boldsymbol{\xi})=\hat{h}_{n}^{(N)}(\xi),
$$

where $\xi=|\boldsymbol{\xi}|$.

The mapping $\mathcal{T S}$ has the trivial fixed point $\hat{h}_{G}(\xi)=\exp \left(-\xi^{2}\right)$.

\subsection{The Lee-Yang property}

Let us introduce a 'potential' $V_{n}^{(N)}(\xi)$ and its Taylor coefficients $\mu_{k, n}^{(N)}$ by

$$
\begin{aligned}
\hat{h}_{n}^{(N)}(\xi) & =e^{-V_{n}^{(N)}(\xi)}, \\
V_{n}^{(N)}(\xi) & =\sum_{k=1}^{\infty} \mu_{k, n}^{(N)} \xi^{k}
\end{aligned}
$$


for $n \geq 0$. (Note that $\hat{h}_{n}^{(N)}(0)=1$, i.e. $V_{n}^{(N)}(0)=0$.) The coefficient $\mu_{k, n}^{(N)}$ is called a truncated correlation. Since $\hat{h}_{n}^{(N)}(\xi)$ is even, $\mu_{k, n}^{(N)}$ vanishes if $k$ is odd.

As is well-known, the hierarchical model has the Lee-Yang property for any $N \geq 1: \hat{h}_{n}^{(N)}(\xi)$ has only real zeros. (See e.g. [8.) As a result, the truncated correlations have the bound [10]:

$$
0 \leq k \mu_{2 k, n}^{(N)} \leq\left(2 \mu_{4, n}^{(N)}\right)^{k / 2}, k \geq 3, n \geq 0
$$

This implies the following:

(1) The Taylor expansion in the right hand side of (2.5) has a nonzero radius of convergence;

(2) It suffices to prove $\lim _{n \rightarrow \infty} \mu_{4, n}^{(N)}=0$ in order to ensure $\lim _{n \rightarrow \infty} \mu_{2 k, n}^{(N)}=0$ for all $k \geq 2$, which implies weak convergence of the trajectory to a Gaussian measure.

Next we introduce the scaled potential $v_{n}^{(N)}(\eta)$ and its Taylor expansion by

$$
v_{n}^{(N)}(\eta)=\frac{1}{N} V_{n}^{(N)}(\sqrt{N} \eta)=\sum_{k=1}^{\infty} \nu_{k, n}^{(N)} \eta^{k}, \quad n \geq 0
$$

In other words, we scale the truncated correlation $\mu_{k, n}^{(N)}$ as

$$
\nu_{k, n}^{(N)}=N^{k / 2-1} \mu_{k, n}^{(N)}, \quad k \geq 1, n \geq 0
$$

Then, $\nu_{k, n}^{(N)}$ turns out to be $\mathcal{O}(1)$ with respect to $N$ (Lemma 5.4). We refer to $\nu_{k, n}^{(N)}$ as a scaled truncated correlation. In particular, for the trivial fixed point measure $h_{G}(\boldsymbol{x})$, the scaled potential is given by

$$
v_{G}(\eta)=\eta^{2}
$$

\subsection{Differential equations for potentials}

In view of (2.2) and (2.3), we consider the following equation:

$$
\frac{\partial}{\partial t} \hat{h}_{n}^{(N)}(t, \boldsymbol{\xi})=-\triangle \hat{h}_{n}^{(N)}(t, \boldsymbol{\xi}), \quad n \geq 1, t \in[0, \beta / 2],
$$

or, equivalently

$$
\frac{\partial}{\partial t} \hat{h}_{n}^{(N)}(t, \xi)=-\frac{\partial^{2}}{\partial \xi^{2}} \hat{h}_{n}^{(N)}(t, \xi)-\frac{N-1}{\xi} \frac{\partial}{\partial \xi} \hat{h}_{n}^{(N)}(t, \xi), \quad n \geq 1, t \in[0, \beta / 2]
$$

with the initial condition

$$
\hat{h}_{n}^{(N)}(0, \xi)=\hat{h}_{n-1}^{(N)}\left(\frac{1}{\sqrt{2 \omega}} \xi\right)^{2}, \quad n \geq 1 .
$$

Then, we have

$$
\hat{h}_{n}^{(N)}(\xi)=\frac{\hat{h}_{n}^{(N)}\left(\frac{\beta}{2}, \xi\right)}{\hat{h}_{n}^{(N)}\left(\frac{\beta}{2}, 0\right)}, \quad n \geq 1 .
$$

We also define the $t$-dependent scaled potential and its expansion by

$$
v_{n}^{(N)}(t, \eta)=-\frac{1}{N} \log \hat{h}_{n}^{(N)}(t, \sqrt{N} \eta)=\sum_{k=1}^{\infty} \nu_{k, n}^{(N)}(t) \eta^{k}, \quad n \geq 1, t \in[0, \beta / 2]
$$


Then, the potentials $v_{n}^{(N)}(t, \eta), n \geq 1$, obey

$$
\begin{aligned}
\frac{\partial}{\partial t} v_{n}^{(N)}(t, \eta) & =\left(\frac{\partial}{\partial \eta} v_{n}^{(N)}(t, \eta)\right)^{2}-\left(1-\frac{1}{N}\right) \frac{1}{\eta} \frac{\partial}{\partial \eta} v_{n}^{(N)}(t, \eta)-\frac{1}{N} \frac{\partial^{2}}{\partial \eta^{2}} v_{n}^{(N)}(t, \eta), \\
v_{n}^{(N)}(0, \eta) & =2 v_{n-1}^{(N)}\left(\frac{1}{\sqrt{2 \omega}} \eta\right) \\
v_{n}^{(N)}(\eta) & =v_{n}^{(N)}\left(\frac{\beta}{2}, \eta\right)-v_{n}^{(N)}\left(\frac{\beta}{2}, 0\right),
\end{aligned}
$$

and the Taylor coefficients $\nu_{2 j, n}^{(N)}(t), j \geq 1, n \geq 1$, obey

$$
\begin{aligned}
\frac{d}{d t} \nu_{2 j, n}^{(N)}(t) & =\sum_{\substack{m+\ell=2 j+2 \\
m, \ell \geq 2}} m \ell \nu_{m, n}^{(N)}(t) \nu_{\ell, n}^{(N)}(t)-(2 j+2)\left(1+\frac{2 j}{N}\right) \nu_{2 j+2, n}^{(N)}(t), \\
\nu_{2 j, n}^{(N)}(0) & =\frac{2}{(2 \omega)^{j}} \nu_{2 j, n-1}^{(N)}, \\
\nu_{2 j, n}^{(N)} & =\nu_{2 j, n}^{(N)}\left(\frac{\beta}{2}\right) .
\end{aligned}
$$

The scaled potential (2.8) is a fixed point of the above recursion relations.

Note that $\nu_{2 j, n}^{(N)}(t)$ has the positivity due to the Lee-Yang property

$$
\nu_{2 j, n}^{(N)}(t) \geq 0, \quad j \geq 1, n \geq 1,
$$

since $\nu_{2 j, n}^{(N)}(t)$ is regarded as a scaled truncated correlation for a hierarchical model with $t$-dependence. (See (5.6)-(5.9) $)$

\subsection{Proof of Theorem 1.1}

In the weak coupling regime, i.e. in the vicinity of the trivial fixed point (2.8), we write $\nu_{k, n}^{(N)}, k=2,6,8$, as follows:

$$
\begin{aligned}
& \nu_{2, n}^{(N)}=1+\frac{1}{\sqrt{2}}\left(1+\frac{2}{N}\right) \nu_{4, n}^{(N)}+\zeta_{2, n}^{(N)} \nu_{4, n}^{(N)^{2}} \\
& \nu_{6, n}^{(N)}=4 \nu_{4, n}^{(N)^{2}}+\zeta_{6, n}^{(N)} \nu_{4, n}^{(N)^{3}} \\
& \nu_{8, n}^{(N)}=\zeta_{8, n}^{(N)} \nu_{4, n}^{(N)^{3}}
\end{aligned}
$$

where $\nu_{4, n}^{(N)}$ is assumed to be small. In fact, analyzing solutions to (2.13)-2.15), we obtain the following proposition (proved at the end of Section 31).

Proposition 2.1 Suppose that there exist a positive integer $n_{1}$ and positive constants $\alpha_{ \pm}\left(\alpha_{-}<\alpha_{+}\right)$ such that

(1) it holds that

$$
\begin{aligned}
& \zeta_{2, n_{1}}^{(N)}=\zeta, \quad \text { if } \alpha=\alpha_{+}, \\
& \zeta_{2, n_{1}}^{(N)}=-\zeta, \quad \text { if } \alpha=\alpha_{-},
\end{aligned}
$$

(2) for $\alpha \in\left[\alpha_{-}, \alpha_{+}\right]$, the following conditions are satisfied:

$$
\begin{aligned}
\left|\zeta_{2, n_{1}}^{(N)}\right| & \leq \zeta, \\
\nu_{4, n_{1}}^{(N)} & \leq \epsilon, \\
\left|\zeta_{6, n_{1}}^{(N)}\right| \nu_{4, n_{1}}^{(N)} & \leq \epsilon_{0}, \\
\left|\zeta_{8, n_{1}}^{(N)}\right| \nu_{4, n_{1}}^{(N)} & \leq \epsilon_{1},
\end{aligned}
$$


where $\zeta, \epsilon, \epsilon_{0}$ and $\epsilon_{1}$ are positive constants determined in Section 3.2. Then, there exists a value $\alpha_{N} \in$ $\left[\alpha_{-}, \alpha_{+}\right]$such that

$$
\begin{aligned}
& \lim _{n \rightarrow \infty} \nu_{2, n}^{(N)}=1, \\
& \lim _{n \rightarrow \infty} \nu_{4, n}^{(N)}=0
\end{aligned}
$$

hold at $\alpha=\alpha_{N}$.

Next we formally put $N=\infty$ in (2.10). Namely, we consider the equation

$$
\frac{\partial}{\partial t} v_{n}^{(\infty)}(t, \eta)=\left(\frac{\partial}{\partial \eta} v_{n}^{(\infty)}(t, \eta)\right)^{2}-\frac{1}{\eta} \frac{\partial}{\partial \eta} v_{n}^{(\infty)}(t, \eta)
$$

with

$$
\begin{aligned}
v_{n}^{(\infty)}(0, \eta) & =2 v_{n-1}^{(\infty)}\left(\frac{1}{\sqrt{2 \omega}} \eta\right), \\
v_{n}^{(\infty)}(\eta) & =v_{n}^{(\infty)}\left(\frac{\beta}{2}, \eta\right)-v_{n}^{(\infty)}\left(\frac{\beta}{2}, 0\right),
\end{aligned}
$$

where the initial point is chosen as follows (see Lemma 4.1):

$$
v_{0}^{(\infty)}(\eta)=\int_{0}^{\eta} \frac{2 \alpha^{2} \eta}{1+\sqrt{1-4 \alpha^{2} \eta^{2}}} d \eta\left(=\lim _{N \rightarrow \infty} v_{0}^{(N)}(\eta)\right) .
$$

In Section 4 we solve (2.24)-2.27). The solution is referred to as the $O(\infty)$ trajectory. As is seen in Section 4 the critical value of $\alpha$ is $\sqrt{2+\sqrt{2}}$ and the critical trajectory tends to the trivial fixed point (2.8) as $n \rightarrow \infty$. (See Lemma 4.4)

Now, consider the Taylor expansion

$$
v_{n}^{(\infty)}(\eta)=\sum_{j=1}^{\infty} \nu_{2 j, n}^{(\infty)} \eta^{2 j}, n \geq 0
$$

and write $\nu_{k, n}^{(\infty)}, k=2,6,8, n \geq 0$ as:

$$
\begin{aligned}
& \nu_{2, n}^{(\infty)}=1+\frac{1}{\sqrt{2}} \nu_{4, n}^{(\infty)}+\zeta_{2, n}^{(\infty)} \nu_{4, n}^{(\infty)^{2}}, \\
& \nu_{6, n}^{(\infty)}=4 \nu_{4, n}^{(\infty)^{2}}+\zeta_{6, n}^{(\infty)} \nu_{4, n}^{(\infty)^{3}}, \\
& \nu_{8, n}^{(\infty)}=\zeta_{8, n}^{(\infty)} \nu_{4, n}^{(\infty)^{3}} .
\end{aligned}
$$

Then we have the following proposition (proved at the end of Section 4).

Proposition 2.2 There exist a positive integer $n_{1}$ and positive constants $\alpha_{++}, \alpha_{--}\left(\alpha_{++}>\alpha_{--}\right)$such that

(1) it holds that

$$
\begin{aligned}
& \zeta_{2, n_{1}}^{(\infty)} \geq 2 \zeta, \quad \text { at } \alpha=\alpha_{++}, \\
& \zeta_{2, n_{1}}^{(\infty)} \leq-2 \zeta, \quad \text { at } \alpha=\alpha_{--},
\end{aligned}
$$

(2) for $\alpha \in\left[\alpha_{--}, \alpha_{++}\right]$, the following conditions are satisfied:

$$
\begin{aligned}
0<\nu_{4, n_{1}}^{(\infty)} & \leq \frac{1}{2} \epsilon, \\
\left|\zeta_{6, n_{1}}^{(\infty)}\right| \nu_{4, n_{1}}^{(\infty)} & \leq \frac{1}{2} \epsilon_{0}, \\
\left|\zeta_{8, n_{1}}^{(\infty)}\right| \nu_{4, n_{1}}^{(\infty)} & \leq \frac{1}{2} \epsilon_{1} .
\end{aligned}
$$


In the above, $\zeta, \epsilon, \epsilon_{0}$ and $\epsilon_{1}$ are the same constants as in Proposition 2.1.

Finally we show that the $O(N)$ trajectory is approximated by the $O(\infty)$ trajectory (proved at the end of Section [5].

Proposition 2.3 For each $j=1,2, \cdots$, and for each $n=0,1,2, \cdots$, it holds that

$$
\lim _{N \rightarrow \infty} \nu_{2 j, n}^{(N)}=\nu_{2 j, n}^{(\infty)}
$$

The convergence is uniform in $\alpha$ on any compact subset of $(0, \infty)$.

This fact is by no means trivial, because (2.10) is a singular perturbation of (2.24), to which the standard theory of differential equations does not apply: note that (2.9) is a diffusion equation in the inverse direction of time. We show Proposition 2.3 by means of $1 / N$ expansion developed in 9 .

Theorem 1.1 readily follows from Proposition 2.1]Proposition 2.2 and Proposition 2.3.

Proof of Theorem [1.1. We first use Proposition[2.2 and fix the integer $n_{1}$. Then using Proposition 2.3 for $n=n_{1}$ and $j \leq 4$, we see, for a sufficiently large $N$, that

$$
\begin{aligned}
& \zeta_{2, n_{1}}^{(N)} \geq \zeta, \quad \text { at } \alpha=\alpha_{++}, \\
& \zeta_{2, n_{1}}^{(N)} \leq-\zeta, \quad \text { at } \alpha=\alpha_{--},
\end{aligned}
$$

and that, for $\alpha \in\left[\alpha_{--}, \alpha_{++}\right]$,

$$
\begin{gathered}
0<\nu_{4, n_{1}}^{(N)} \leq \epsilon, \\
\left|\zeta_{6, n_{1}}^{(N)}\right| \nu_{4, n_{1}}^{(N)} \leq \epsilon_{0}, \\
\left|\zeta_{8, n_{1}}^{(N)}\right| \nu_{4, n_{1}}^{(N)} \leq \epsilon_{1} .
\end{gathered}
$$

Since $\zeta_{2, n_{1}}^{(N)}$ is continuous with respect to $\alpha \in\left[\alpha_{--}, \alpha_{++}\right]$, we can choose a subinterval $\left[\alpha_{-}, \alpha_{+}\right] \subset$ $\left[\alpha_{--}, \alpha_{++}\right]$so that the assumptions of Proposition 2.1 are satisfied. Theorem [1.1] follows from (2.22) and (2.23) by virtue of (2.6).

\section{$3 O(N)$ trajectory in weak coupling regime}

In this section, we analyze the solution to (2.13)-2.15) in the weak coupling regime and prove Proposition 2.1 We shall abbreviate $\nu_{k, n}^{(N)}(t), \nu_{k, n}^{(N)}$ and $\zeta_{k, n}^{(N)}$ as $\nu_{k, n}(t), \nu_{k, n}$ and $\zeta_{k, n}$, respectively, since we fix $N$ throughout this section. All the bounds in this section are uniform in $N$.

\subsection{Recursion}

Let us consider (2.13)- 2.15). We introduce functions $\lambda_{2 j, n}(\tau), j \geq 1, n \geq 1$, by

$$
\nu_{2 j, n}(t)=\sigma(t) \omega^{-1} \nu_{2, n-1} \delta_{j, 1}+\sigma(t)^{2 j} \lambda_{2 j, n}(\sigma(t) t), \quad j \geq 1, n \geq 1,
$$

in order to separate the main contribution $\sigma(t) \omega^{-1} \nu_{2, n-1}$ to the 'mass term' $\nu_{2, n}(t)$, where

$$
\sigma(t)=\frac{1}{1-4 \omega^{-1} \nu_{2, n-1} t} .
$$

In what follows, we assume that $\sigma(t)$ is defined for $t \in[0, \beta / 2]=[0,(\omega-1) / 4]$. This is the case if $\nu_{2, n-1}$ is close to 1 . 
It is easily seen that $\lambda_{2 j, n}(\tau), j \geq 1$, satisfy the same equations as those for $\nu_{2 j, n}^{(N)}(t)$ :

$$
\frac{d}{d \tau} \lambda_{2 j, n}(\tau)=\sum_{\substack{m+\ell=2 j+2 \\ m, \ell \geq 2}} m \ell \lambda_{m, n}(\tau) \lambda_{\ell, n}(\tau)-(2 j+2)\left(1+\frac{2 j}{N}\right) \lambda_{2 j+2, n}(\tau), \quad j \geq 1,
$$

with

$$
\begin{aligned}
\lambda_{2 j, n}(0) & =\left\{\begin{array}{cl}
0, & j=1, \\
\frac{2}{(2 \omega)^{j}} \nu_{2 j, n-1}, & j \geq 2,
\end{array}\right. \\
\nu_{2 j, n} & =r \nu_{2, n-1} \delta_{j, 1}+(r \omega)^{2 j} \lambda_{2 j, n}(T), \quad j \geq 1,
\end{aligned}
$$

where

$$
\begin{aligned}
r & =\frac{1}{1-(\omega-1)\left(\nu_{2, n-1}-1\right)}, \\
T & =\frac{1}{4} \omega(\omega-1) r .
\end{aligned}
$$

Let us rewrite (3.1) and (3.2) for $j \leq 4$ as integral equations:

$$
\begin{aligned}
& \lambda_{2, n}(\tau)=\int_{0}^{\tau}\left(4 \lambda_{2, n}(\tau)^{2}-4\left(1+\frac{2}{N}\right) \lambda_{4, n}(\tau)\right) d \tau \\
& \lambda_{4, n}(\tau)=\frac{1}{2 \omega^{2}} \nu_{4, n-1}+\int_{0}^{\tau}\left(16 \lambda_{2, n}(\tau) \lambda_{4, n}(\tau)-6\left(1+\frac{4}{N}\right) \lambda_{6, n}(\tau)\right) d \tau \\
& \lambda_{6, n}(\tau)=\frac{1}{4 \omega^{3}} \nu_{6, n-1}+\int_{0}^{\tau}\left(24 \lambda_{2, n}(\tau) \lambda_{6, n}(\tau)+16 \lambda_{4, n}(\tau)^{2}-8\left(1+\frac{6}{N}\right) \lambda_{8, n}(\tau)\right) d \tau \\
& \lambda_{8, n}(\tau)=\frac{1}{8 \omega^{4}} \nu_{8, n-1}+\int_{0}^{\tau}\left(32 \lambda_{2, n}(\tau) \lambda_{8, n}(\tau)+48 \lambda_{4, n}(\tau) \lambda_{6, n}(\tau)-10\left(1+\frac{8}{N}\right) \lambda_{10, n}(\tau)\right) d \tau
\end{aligned}
$$

We now derive expressions for $\zeta_{k, n}, j=2,6,8$, introduced in (2.17)-2.19) and confirm the 'marginal behavior' of $\nu_{4, n}$ by using $\omega=\sqrt{2}(d=4)$. Successive use of (3.6)-3.9) yields

$$
\begin{aligned}
& \lambda_{2, n}(\tau)=-4\left(1+\frac{2}{N}\right) \frac{1}{4} \nu_{4, n-1} \tau+X_{2}(\tau), \\
& \lambda_{4, n}(\tau)=\frac{1}{4} \nu_{4, n-1}+X_{4}(\tau), \\
& \lambda_{6, n}(\tau)=\frac{1}{8 \sqrt{2}} \nu_{6, n-1}+\nu_{4, n-1}^{2} \tau+X_{6}(\tau), \\
& \lambda_{8, n}(\tau)=\frac{1}{32} \nu_{8, n-1}+X_{8}(\tau),
\end{aligned}
$$

where

$$
\begin{aligned}
& X_{2}(\tau)=\int_{0}^{\tau}\left(4 \lambda_{2, n}(\tau)^{2}-4\left(1+\frac{2}{N}\right) X_{4}(\tau)\right) d \tau \\
& X_{4}(\tau)=\int_{0}^{\tau}\left(16 \lambda_{2, n}(\tau) \lambda_{4, n}(\tau)-6\left(1+\frac{4}{N}\right) \lambda_{6, n}(\tau)\right) d \tau \\
& X_{6}(\tau)=\int_{0}^{\tau}\left(24 \lambda_{2, n}(\tau) \lambda_{6, n}(\tau)-8 \nu_{4, n-1} X_{4}(\tau)+16 X_{4}(\tau)^{2}-8\left(1+\frac{6}{N}\right) \lambda_{8, n}(\tau)\right) d \tau \\
& X_{8}(\tau)=\int_{0}^{\tau}\left(32 \lambda_{2, n}(\tau) \lambda_{8, n}(\tau)+48 \lambda_{4, n}(\tau) \lambda_{6, n}(\tau)-10\left(1+\frac{8}{N}\right) \lambda_{10, n}(\tau)\right) d \tau
\end{aligned}
$$


Using the above expressions, we obtain the following recursion relations:

$$
\begin{aligned}
\zeta_{2, n} \nu_{4, n}^{2} & =\sqrt{2} r \zeta_{2, n-1} \nu_{4, n-1}^{2}+Y_{2}, \\
\zeta_{6, n} \nu_{4, n}^{3} & =\frac{1}{\sqrt{2}} r^{6} \zeta_{6, n-1} \nu_{4, n-1}^{3}+Y_{6}, \\
\zeta_{8, n} \nu_{4, n}^{3} & =\frac{1}{2} r^{8} \zeta_{8, n-1} \nu_{4, n-1}^{3}+Y_{8}, \\
\nu_{4, n} & =\nu_{4, n-1}-\left(\frac{1}{2}+\frac{4}{N}\right) \nu_{4, n-1}^{2}+Y_{4},
\end{aligned}
$$

where

$$
\begin{gathered}
Y_{2}=\frac{1}{\sqrt{2}}\left(1+\frac{2}{N}\right)\left(\nu_{4, n-1}-\nu_{4, n}\right)+\frac{r-1}{\sqrt{2}}\left(1+\frac{2}{N}\right) \nu_{4, n-1} \\
\quad+\left(1-\frac{1}{\sqrt{2}}\right)\left(r-r^{3}\right)\left(1+\frac{2}{N}\right) \nu_{4, n-1}+2 r^{2} X_{2}(T) \\
Y_{4}=-(3-2 \sqrt{2})\left(1+\frac{2}{N}\right)\left(r^{6}-1\right) \nu_{4, n-1}^{2}-3(\sqrt{2}-1)\left(1+\frac{4}{N}\right)\left(r^{5}-1\right) \nu_{4, n-1}^{2} \\
\quad-\frac{3(3-2 \sqrt{2})}{2}\left(1+\frac{4}{N}\right)\left(r^{6}-1\right) \nu_{4, n-1}^{2}+(4-2 \sqrt{2})\left(1+\frac{2}{N}\right)(r-1) \nu_{4, n-1}^{2} \\
\quad+4(\sqrt{2}-1) r \zeta_{2, n-1} \nu_{4, n-1}^{3}+(r-1)^{2}\left(r^{2}+2 r+3\right) \nu_{4, n-1}+4 r^{4} X_{4}(T) \\
\quad-64\left(1+\frac{2}{N}\right) r^{4} \nu_{4, n-1} \int_{0}^{T} \tau X_{4}(\tau) d \tau+64 r^{4} \int_{0}^{T} X_{2}(\tau) \lambda_{4, n}(\tau) d \tau \\
\quad-\frac{3(\sqrt{2}-1)}{4}\left(1+\frac{4}{N}\right) r^{5} \zeta_{6, n-1} \nu_{4, n-1}^{3}-24\left(1+\frac{4}{N}\right) r^{4} \int_{0}^{T} X_{6}(\tau) d \tau, \\
Y_{6}=4\left(\nu_{4, n-1}^{2}-\nu_{4, n}^{2}\right)+2 \sqrt{2}\left(r^{6}-1\right) \nu_{4, n-1}^{2}+(4-2 \sqrt{2})\left(r^{7}-1\right) \nu_{4, n-1}^{2}+8 r^{6} X_{6}(T), \\
Y_{8}=16 r^{8} X_{8}(T) .
\end{gathered}
$$

\subsection{Bounds}

Let us derive bounds on $\zeta_{2 j, n}, j=1,3,4$, and $\nu_{4, n}$ by means of (3.14)-(3.17). Our starting point is:

$$
\begin{aligned}
& \lambda_{k, n}(\tau) \geq 0, \quad k=4,6,8,10, \\
& \lambda_{2, n}(\tau) \leq 0 .
\end{aligned}
$$

The first inequality comes from (2.16). The second one is shown as follows. From (2.13), we see that $\nu_{2, n}(t)$ obeys

$$
\frac{d}{d t} \nu_{2, n}(t)=4 \nu_{2, n}(t)^{2}-4\left(1+\frac{2}{N}\right) \nu_{4, n}(t)
$$

whereas the function $\tilde{\nu}_{2, n}(t)=\sigma(t) \omega^{-1} \nu_{2, n-1}$ satisfies

$$
\frac{d}{d t} \tilde{\nu}_{2, n}(t)=4 \tilde{\nu}_{2, n}(t)^{2}
$$

Since $\nu_{2, n}(0)=\tilde{\nu}_{2, n}(0)$ and $\nu_{4, n}(t) \geq 0$, we have

$$
\nu_{2, n}(t) \leq \tilde{\nu}_{2, n}(t)
$$

and hence (3.23).

Using (3.22) and (3.23), we obtain the following lemma.

Lemma 3.1 Suppose that $\nu_{4, n-1}$ and $\zeta_{m, n-1}, m=2,6,8$, satisfy

$$
\begin{aligned}
\nu_{4, n-1} & \leq \epsilon_{0}, \\
\left|\zeta_{m, n-1}\right| \nu_{4, n-1} & \leq \epsilon_{0}, \quad m=2,6,8 .
\end{aligned}
$$


Then $\nu_{4, n}$ and $\zeta_{m, n}, m=2,6,8$, satisfy

$$
\begin{aligned}
\nu_{4, n-1}-5 \nu_{4, n-1}^{2} & <\nu_{4, n}<\nu_{4, n-1}-\frac{1}{4} \nu_{4, n-1}^{2}, \\
\left|\zeta_{2, n}-\sqrt{2} \zeta_{2, n-1}\right| & <C_{1} \\
\left|\zeta_{6, n}\right| & <0.8\left|\zeta_{6, n-1}\right|+C_{2}\left(1+\zeta_{8, n-1}\right), \\
\left|\zeta_{8, n}\right| & <0.6\left|\zeta_{8, n-1}\right|+C_{3}
\end{aligned}
$$

where $C_{1}, C_{2}, C_{3}$ and $\epsilon_{0}$ are positive constants independent of $N, n$ and $\alpha$.

Proof. Suppose that $\nu_{k, n-1}, k=2,6,8$, have the following forms

$$
\begin{aligned}
& \nu_{2, n-1}=1+\frac{1}{\sqrt{2}}\left(1+\frac{2}{N}\right) \nu_{4, n-1}+\zeta_{2, n-1} \nu_{4, n-1}^{2}, \\
& \nu_{6, n-1}=4 \nu_{4, n-1}^{2}+\zeta_{6, n-1} \nu_{4, n-1}^{3}, \\
& \nu_{8, n-1}=\zeta_{8, n-1} \nu_{4, n-1}^{3}
\end{aligned}
$$

with the bounds (3.24) and (3.25). Then, if we choose $\epsilon_{0}$ sufficiently small, equations (3.4)-(3.8) together with (3.22) and (3.23) yield

$$
\begin{aligned}
& 1<r<1+5 \nu_{4, n-1}, \\
& 0<T<\frac{1}{5} \\
& -\frac{3}{5} \nu_{4, n-1} \leq \lambda_{2, n}(\tau) \leq 0, \\
& 0 \leq \lambda_{4, n}(\tau) \leq \frac{1}{4} \nu_{4, n-1}, \\
& 0 \leq \lambda_{6, n}(\tau) \leq \nu_{4, n-1}^{2} .
\end{aligned}
$$

Using the above bounds, we have for $\tau \in[0, T]$

$$
\begin{aligned}
\left|X_{2}(\tau)\right| & \leq \text { const. } \nu_{4, n-1}^{2}, \\
\left|X_{4}(\tau)\right| & \leq \text { const. } \nu_{4, n-1}^{2}, \\
\left|X_{6}(\tau)\right| & \leq \text { const. }\left(1+\zeta_{8, n-1}\right) \nu_{4, n-1}^{3}, \\
X_{8}(\tau) & \leq \text { const. } \nu_{4, n-1}^{3},
\end{aligned}
$$

and hence

$$
\begin{aligned}
\left|Y_{2}\right| & \leq \text { const. } \nu_{4, n-1}^{2} \\
\left|Y_{4}\right| & \leq \text { const. }\left(1+\left|\zeta_{2, n-1}\right|+\left|\zeta_{6, n-1}\right|+\left|\zeta_{8, n-1}\right|\right) \nu_{4, n-1}^{3} \\
\left|Y_{6}\right| & \leq \text { const. }\left(1+\left|\zeta_{8, n-1}\right|\right) \nu_{4, n-1}^{3}, \\
Y_{8} & \leq \text { const. } \nu_{4, n-1}^{3} .
\end{aligned}
$$

The lemma follows from the above bounds and (3.14)-(3.17) combined with the assumptions (3.24) and (3.25), if we choose appropriate constants $C_{1}, C_{2}$ and $C_{3}$.

Remark. The function $\sigma(t)$ is well-defined on $[0, \beta / 2]$ under (3.24) and (3.25), if we choose $\epsilon_{0}$ sufficiently small.

Using the positive constants $C_{1}, C_{2}, C_{3}$ and $\epsilon_{0}$ in Lemma 3.1 we put

$$
\begin{aligned}
\zeta & =(\sqrt{2}+1) C_{1}, \\
\epsilon_{1} & =\min \left(\epsilon_{0}, \frac{\epsilon_{0}}{10 C_{2}}\right), \\
\epsilon & =\min \left(\epsilon_{0}, \frac{\epsilon_{0}}{\zeta}, \frac{\epsilon_{0}}{10 C_{2}}, \frac{2 \epsilon_{1}}{5 C_{3}}\right) .
\end{aligned}
$$


Proof of Proposition [2.1. Put $A=\left[\alpha_{-}, \alpha_{+}\right]$. Under the assumption (2) of Proposition 2.1 we see that (3.24) and (3.25) are satisfied for $n=n_{1}+1$ and for any $\alpha \in A$. From the assumption (1) and (3.27), we have

$$
\begin{array}{ll}
\zeta_{2, n_{1}+1}>\zeta & \text { at } \alpha=\alpha_{+}, \\
\zeta_{2, n_{1}+1}<-\zeta & \text { at } \alpha=\alpha_{-} .
\end{array}
$$

These bounds imply that $\zeta_{2, n_{1}+1}$ runs through $[-\zeta, \zeta]$ when $\alpha$ scans $A$. Then, we can find a subinterval $A^{\prime}=\left[\alpha_{-}^{\prime}, \alpha_{+}^{\prime}\right]$ of $A$ such that

$$
\begin{aligned}
\zeta_{2, n_{1}+1} & = \pm \zeta & & \text { at } \alpha=\alpha_{ \pm}^{\prime}, \\
\left|\zeta_{2, n_{1}+1}\right| & \leq \zeta & & \text { for } \alpha \in A^{\prime} .
\end{aligned}
$$

Furthermore, from (3.26), (3.28), (3.29) and the assumption (2) of Proposition 2.1] we have

$$
\begin{aligned}
\nu_{4, n_{1}+1} & <\nu_{4, n_{1}}-\frac{1}{4} \nu_{4, n_{1}}^{2}, \\
\left|\zeta_{6, n_{1}+1}\right| \nu_{4, n_{1}+1} & <\epsilon_{0} \\
\left|\zeta_{8, n_{1}+1}\right| \nu_{4, n_{1}+1} & <\epsilon_{1}
\end{aligned}
$$

for any $\alpha \in A$. Thus, the assumptions of Proposition 2.1 have been reproduced at the next stage by replacing $\alpha_{ \pm}$by $\alpha_{ \pm}^{\prime}$. Therefore, Bleher-Sinai argument [1, 2, 11] [7, P.25] applies and, (2.22) and (2.23) hold for some $\alpha \in A$ because of (3.37) and 2.17).

\section{$4 O(\infty)$ trajectory}

In this section, we solve the recursion relations (2.24) -(2.26) with (2.27). Using the solution, we derive bounds on the Taylor coefficients $\nu_{2 j, n}^{(\infty)}, j=1,2,3,4$, defined in (2.28) and show Proposition 2.2

\subsection{Recursions}

We firstly confirm the initial point (2.27).

Lemma 4.1 Put

$$
D_{\theta}=\left\{(\alpha, \eta) \in(0, \infty) \times \mathbb{C}|2 \alpha| \eta \mid<1 \text { or }\left|\arg \eta-\frac{\pi}{2}\right|<\theta \text { or }\left|\arg \eta+\frac{\pi}{2}\right|<\theta\right\}
$$

for $\theta>0$. Then, for a sufficiently small $\theta$, it holds that

$$
\lim _{N \rightarrow \infty} \frac{d}{d \eta} v_{0}^{(N)}(\eta)=\frac{2 \alpha^{2} \eta}{1+\sqrt{1-4 \alpha^{2} \eta^{2}}}, \quad(\alpha, \eta) \in D_{\theta},
$$

where the convergence is uniform in $(\alpha, \eta)$ on any compact subset of $D_{\theta}$. Furthermore, $\nu_{2 j, 0}^{(N)}, j \geq 1$, converges as $N \rightarrow \infty$.

Remark. In this section, we use (4.1) only for $\eta$ with $2 \alpha|\eta|<1$. The result for $\eta$ near the imaginary axis will be used in Section [

Proof. The Fourier transform of (1.6) is written as

$$
\hat{h}_{0}^{(N)}(\xi)=\Gamma(N / 2) \frac{J_{N / 2-1}(\sqrt{N} \alpha \xi)}{(\sqrt{N} \alpha \xi / 2)^{N / 2-1}}
$$


as a function of $\xi=|\boldsymbol{\xi}|$, where $J_{\nu}$ denotes the Bessel function of order $\nu$ :

$$
J_{\nu}(z)=\frac{\left(\frac{z}{2}\right)^{\nu}}{\sqrt{\pi} \Gamma\left(\nu+\frac{1}{2}\right)} \int_{0}^{\pi} \cos (z \cos \theta) \sin ^{2 \nu} \theta d \theta .
$$

The derivative of the scaled potential defined by (2.7) is therefore given by

$$
\frac{d}{d \eta} v_{0}^{(N)}(\eta)=-\frac{1}{N} \frac{\frac{d}{d \eta} \hat{h}_{0}^{(N)}(\sqrt{N} \eta)}{\hat{h}_{0}^{(N)}(\sqrt{N} \eta)}=\alpha \frac{J_{N / 2}(N \alpha \eta)}{J_{N / 2-1}(N \alpha \eta)}
$$

Then, using the relation

$$
J_{\nu-1}(z)+J_{\nu+1}(z)=\frac{2 \nu}{z} J_{\nu}(z)
$$

we have

$$
\eta \frac{d}{d \eta} v_{0}^{(N)}(\eta)=\frac{c_{0}}{1-\frac{c_{1}}{1-\frac{c_{2}}{1-\frac{c_{3}}{1-\cdot} \cdot \frac{c_{\ell-1}}{1-w_{\ell}}}}}, \quad \ell \geq 1
$$

where

$$
\begin{aligned}
c_{0} & =\alpha^{2} \eta^{2}, \\
c_{j} & =\frac{\alpha^{2} \eta^{2}}{\left(1+\frac{2(j-1)}{N}\right)\left(1+\frac{2 j}{N}\right)}, \quad j \geq 1, \\
w_{\ell} & =\frac{\alpha \eta}{1+\frac{2(\ell-1)}{N}} \frac{J_{N / 2+\ell}(\alpha N \eta)}{J_{N / 2+\ell-1}(\alpha N \eta)}, \quad \ell \geq 1 .
\end{aligned}
$$

Now, let $\theta$ be a sufficiently small positive constant, and let $D^{\prime}$ be any compact subset of $D_{\theta}$. Then, by choosing a sufficiently large $\ell$ (depending on $D^{\prime}$ ), we see that the right hand side of (4.5) is well-defined for $(\alpha, \eta) \in D^{\prime}$ and is holomorphic in $\eta$. Furthermore, it holds that

$$
\lim _{N \rightarrow \infty} \eta \frac{d}{d \eta} v_{0}^{(N)}(\eta)=\frac{\alpha^{2} \eta^{2}}{1-\frac{\alpha^{2} \eta^{2}}{1-\frac{\alpha^{2} \eta^{2}}{1-\frac{\alpha^{2} \eta^{2}}{1-\cdot}}}}=\frac{2 \alpha^{2} \eta^{2}}{1+\sqrt{1-4 \alpha^{2} \eta^{2}}}
$$

for $(\alpha, \eta) \in D^{\prime}$, where the convergence is uniform on $D^{\prime}$. The last statement of the lemma is obvious.

Consider the recursion relations (2.24)-2.27). Since $v_{n}^{(\infty)}(t, \eta)$ and $v_{n}^{(\infty)}(\eta)$ are even with respect to $\eta$, we can define functions $u_{n}(t, x)$ and $u_{n}(x)$ by

$$
\begin{gathered}
u_{n}\left(t, \eta^{2}\right)=v_{n}^{(\infty)}(t, \eta), n \geq 1, \\
u_{n}\left(\eta^{2}\right)=v_{n}^{(\infty)}(\eta), n \geq 0
\end{gathered}
$$


respectively. Then, (2.24) (2.27) become

$$
\begin{aligned}
\frac{\partial}{\partial t} u_{n}(t, x) & =4 x\left(\frac{\partial}{\partial x} u_{n}(t, x)\right)^{2}-2 \frac{\partial}{\partial x} u_{n}(t, x), \\
u_{n}(0, x) & =2 u_{n-1}\left(\frac{x}{2 \omega}\right), \\
u_{n}(x) & =u_{n}\left(\frac{\beta}{2}, x\right)-u_{n}\left(\frac{\beta}{2}, 0\right), \\
u_{0}(x) & =\int_{0}^{x} \frac{\alpha^{2}}{1+\sqrt{1-4 \alpha^{2} y}} d y,
\end{aligned}
$$

where $n \geq 1$.

Now, let us denote the inverse of $p=u_{n}^{\prime}(x)$ by $x=w_{n}(p)$ and the inverse of $p=u_{n}^{\prime}(t, x)$ by $x=w_{n}(t, p)$ for each $t$. Then, $w_{n}(p)$ and $w_{n}(t, p)$ obey the following recursion relations:

$$
\begin{aligned}
\frac{\partial w_{n}}{\partial t}(t, p) & =-4 p^{2} \frac{\partial w_{n}}{\partial p}(t, p)-8 p w_{n}(t, p)+2, \\
w_{n}(0, p) & =2 \omega w_{n-1}(\omega p) \\
w_{n}(p) & =w_{n}\left(\frac{\beta}{2}, p\right) \\
w_{0}(p) & =\frac{1}{2 p}-\frac{\alpha^{2}}{4 p^{2}} .
\end{aligned}
$$

\subsection{Solutions}

The equation (4.16) with the initial condition (4.17) can be explicitly solved. In fact, as is checked by direct calculation, the function

$$
w_{n}(t, p)=\frac{1}{2 p^{2}}\left(p-\frac{1}{p^{-1}+4 t}+\frac{4 \omega}{\left(p^{-1}+4 t\right)^{2}} w_{n-1}\left(\frac{\omega}{p^{-1}+4 t}\right)\right)
$$

solves (4.16) and (4.17). This together with (4.18) and (1.7) implies

$$
w_{n}(p)=\frac{1}{2 p^{2}}\left(p-\frac{1}{p^{-1}+\omega-1}+\frac{4 \omega}{\left(p^{-1}+\omega-1\right)^{2}} w_{n-1}\left(\frac{\omega}{p^{-1}+\omega-1}\right)\right) .
$$

This recursion with (4.19) is then solved as follows:

$$
w_{n}(p)=\frac{1}{2 p^{2}}\left(p+\frac{1}{2} \sum_{j=1}^{n} \frac{2^{j}}{\omega^{j}-1+p^{-1}}-\frac{1}{2}\left(\frac{2}{\omega}\right)^{n} \alpha^{2}\right), \quad n \geq 1, p>0 .
$$

Furthermore, this combined with (4.20) yields

$$
w_{n}(t, p)=\frac{1}{2 p^{2}}\left(p+\frac{1}{2} \sum_{j=1}^{n} \frac{2^{j}}{\omega^{j}-\omega+4 t+p^{-1}}-\frac{1}{2}\left(\frac{2}{\omega}\right)^{n} \alpha^{2}\right), \quad n \geq 1, t \geq 0, p>0 .
$$

Now, $u_{n}^{\prime}(t, x)$ is obtained as the inverse of $x=w_{n}(t, p)$. We here have to choose an appropriate branch of the multi-valued inverse.

Lemma 4.2 (1) For $n \geq 1$ and $t \geq 0$, the equation

$$
w_{n}(t, p)=0, p>0
$$

has a unique solution $p$, which we hereafter denote by $\pi_{n}(t)$. The function $\pi_{n}(t)$ is continuous with respect to $t \geq 0$ and satisfies

$$
\frac{\partial w_{n}}{\partial p}\left(t, \pi_{n}(t)\right)>0, \quad t \geq 0 .
$$


(2) For $n \geq 1$, there exists uniquely a continuous function $u_{n}^{\prime}(t, x)$ defined on $\{(t, x) \in \mathbb{R} \times \mathbb{C} \mid t \in$ $\left.\left[0, \frac{\beta}{2}\right],|x|<\rho_{n}\right\}$ such that

$$
\begin{aligned}
w_{n}\left(t, u_{n}^{\prime}(t, x)\right) & =x, \quad t \in\left[0, \frac{\beta}{2}\right],|x|<\rho_{n}, \\
u_{n}^{\prime}(t, 0) & =\pi_{n}(t), \quad t \in\left[0, \frac{\beta}{2}\right],
\end{aligned}
$$

where $\rho_{n}>0$ is a suitably chosen constant independent of $t$. Moreover, the functions $u_{n}^{\prime}(t, x), n \geq 1$, are holomorphic with respect to $x$ and satisfy

$$
\omega u_{n+1}^{\prime}(0,2 \omega x)=u_{n}^{\prime}\left(\frac{\beta}{2}, x\right), \quad n \geq 1,|x|<\min \left(\rho_{n}, \frac{\rho_{n+1}}{2 \omega}\right),
$$

Proof. (1) We see from (4.23) that $2 p^{2} w_{n}(t, p)$ is increasing with respect to $p>0$ and

$$
2 p^{2} w_{n}(t, p) \rightarrow \begin{cases}-\frac{1}{2}\left(\frac{2}{\omega}\right)^{n} \alpha^{2}, & p \rightarrow 0, \\ +\infty, & p \rightarrow+\infty,\end{cases}
$$

holds. Then, (4.24) has a unique solution $p$. The remaining statements are obvious.

(2) Because of (4.25), there exists uniquely a continuous function $p=u_{n}^{\prime}(t, x)$ defined on $\{(t, x) \in$ $\left.\mathbb{R} \times \mathbb{C}\left|t \in\left[0, \frac{\beta}{2}\right],\right| x \mid<\rho_{n}\right\}$ satisfying (4.26) and (4.27) for some positive constant $\rho_{n}$ independent of $t$. Since the function $w_{n}(t, p)$ is holomorphic on the complex half plane $\Re p>0$ for each $t \in\left[0, \frac{\beta}{2}\right]$, $u_{n}^{\prime}(t, x)$ is holomorphic with respect to $x$. Let us show (4.28). Since (4.26), (4.17) and (4.18) imply

$$
x=w_{n}\left(0, u_{n}^{\prime}(0, x)\right)=2 \omega w_{n-1}\left(\omega u_{n}^{\prime}(0, x)\right)=2 \omega w_{n-1}\left(\frac{\beta}{2}, \omega u_{n}^{\prime}(0, x)\right),
$$

we have

$$
w_{n-1}\left(\frac{\beta}{2}, \omega u_{n}^{\prime}(0,2 \omega x)\right)=x .
$$

Furthermore, since (4.17) and (4.18) imply

$$
0=w_{n-1}\left(\frac{\beta}{2}, \pi_{n-1}\left(\frac{\beta}{2}\right)\right)=w_{n-1}\left(\pi_{n-1}\left(\frac{\beta}{2}\right)\right)=\frac{1}{2 \omega} w_{n}\left(0, \frac{1}{\omega} \pi_{n-1}\left(\frac{\beta}{2}\right)\right),
$$

we have

$$
\frac{1}{\omega} \pi_{n-1}\left(\frac{\beta}{2}\right)=\pi_{n}(0)
$$

that is,

$$
\omega u_{n}^{\prime}(0,0)=\omega \pi_{n}(0)=\pi_{n-1}\left(\frac{\beta}{2}\right) .
$$

Therefore, we can conclude from (4.29) that

$$
\omega u_{n}^{\prime}(0,2 \omega x)=u_{n-1}^{\prime}\left(\frac{\beta}{2}, x\right)
$$

holds because of the uniqueness of $u_{n-1}^{\prime}(\beta / 2, x)$.

Using the function $u_{n}^{\prime}(t, x)$ in Lemma 4.2 we define $u_{n}(t, x)$ by

$$
u_{n}(t, x)=\int_{0}^{x} u_{n}^{\prime}(t, y) d y-2 \int_{0}^{t} u_{n}^{\prime}(s, 0) d s
$$

for $n \geq 1, t \in\left[0, \frac{\beta}{2}\right]$ and for $x \in \mathbb{C}$ with $|x|<\rho_{n}$. Obviously, it holds that

$$
u_{n}^{\prime}(t, x)=\frac{\partial}{\partial x} u_{n}(t, x) .
$$

We also define $u_{n}(x), n \geq 0$, so that (4.13) holds. 
Lemma 4.3 The functions $u_{n}(t, x), n \geq 1$, and $u_{n}(x), n \geq 0$, are holomorphic with respect to $x$ and satisfy (4.12) - (4.15). Furthermore, $p=u_{n}^{\prime}(x)=\frac{d}{d x} u_{n}(x)$ is an inverse of $x=w_{n}(p)$.

Proof. Equations (4.16) and (4.26) imply

$$
\frac{\partial}{\partial t} u_{n}^{\prime}(t, x)=8 x u_{n}^{\prime}(t, x) \frac{\partial}{\partial x} u_{n}^{\prime}(t, x)+4 u_{n}^{\prime}(t, x)^{2}-2 \frac{\partial}{\partial x} u_{n}^{\prime}(t, x) .
$$

Therefore, $u_{n}(t, x)$ satisfies (4.12) because of (4.30) and (4.31). Furthermore, (4.13) and (4.28) imply

$$
\frac{\partial}{\partial x} u_{n}(x)=\frac{\partial}{\partial x} u_{n}\left(\frac{\beta}{2}, x\right),
$$

from which we obtain (4.14).

Next, we show (4.15). From (4.13) with $n=1$, we see that

$$
u_{0}^{\prime}(x)=\omega u_{1}^{\prime}(0,2 \omega x) .
$$

Then, (4.26) and (4.17) imply

$$
x=\frac{1}{2 \omega} w_{1}\left(0, u_{1}^{\prime}(0,2 \omega x)\right)=w_{0}\left(\omega u_{1}^{\prime}(0,2 \omega x)\right)=w_{0}\left(u_{0}^{\prime}(x)\right) .
$$

Because of (4.19), this means

$$
x=\frac{1}{2 u_{0}^{\prime}(x)}-\frac{\alpha^{2}}{4 u_{0}^{\prime}(x)^{2}} .
$$

Since (4.34) holds at $x=0$ as well, we have (4.15).

Finally, the function $p=u_{n}^{\prime}(x)$ is an inverse of $x=w_{n}(p)$, because $u_{n}^{\prime}(x)=u_{n}^{\prime}(\beta / 2, x)$ and $w_{n}(p)=$ $w_{n}(\beta / 2, p)$.

Let us define $v_{n}^{(\infty)}(t, \eta)$ and $v_{n}^{(\infty)}(\eta)$ by (4.10) and 4.11), respectively. As a consequence of Lemma4.3. $v_{n}^{(\infty)}(\eta)$ and $v_{n}^{(\infty)}(t, \eta)$ can be Taylor-expanded as (2.28) and

$$
v_{n}^{(\infty)}(t, \eta)=\sum_{j=1}^{\infty} \nu_{2 j, n}^{(\infty)}(t) \eta^{2 j},
$$

respectively, in a neighborhood of $\eta=0$. Thus, in view of (2.24) $-(2.26)$ and (2.27), we see that $\nu_{2 j, n}^{(\infty)}(t)$ and $\nu_{2 j, n}^{(\infty)}$ satisfy the following recursion relations:

$$
\begin{aligned}
\frac{d}{d t} \nu_{2 j}^{(\infty)}(t) & =\sum_{\substack{m+\ell=2 j+2 \\
m, \ell \geq 2}} m \ell \nu_{m, n}^{(\infty)}(t) \nu_{\ell, n}^{(\infty)}(t)-(2 j+2) \nu_{2 j+2, n}^{(\infty)}(t) \\
\nu_{2 j, n}^{(\infty)}(0) & =\frac{2}{(2 \omega)^{j}} \nu_{2 j, n-1}^{(\infty)} \\
\nu_{2 j, n}^{(\infty)} & =\nu_{2 j, n}^{(\infty)}\left(\frac{\beta}{2}\right) \\
\nu_{2 j, 0}^{(\infty)} & =\lim _{N \rightarrow \infty} \nu_{2 j, 0}^{(N)}
\end{aligned}
$$

\subsection{Asymptotics}

In what follows, we derive the asymptotic forms (2.29) - 2.31) with bounds on $\zeta_{k, n}^{(\infty)}, k=2,6,8$, and show Proposition 2.2

As is shown in Lemma $4.3 p=u_{n}^{\prime}(x)$ is an inverse of $x=w_{n}(p)$. Then, the Taylor coefficient

$$
\nu_{2 j, n}^{(\infty)}=\frac{1}{j !} \frac{d^{j-1} p}{d x^{j-1}}(0), \quad j \geq 1, n \geq 1,
$$


is calculated from (4.22). For convenience' sake, we introduce the variable $s$ by

$$
p=\frac{1}{1-s}
$$

and regard $s$ as a function of $x$.

Lemma 4.4 The functions $s=s(x)$ and $p=p(x)$ satisfy

$$
s=\delta_{n}\left(2 x p^{2}+\gamma_{n}-R_{n}(s)\right)
$$

where

$$
\begin{aligned}
\delta_{n} & =\frac{1}{n / 2+1}, \\
\gamma_{n} & =\frac{1}{\sqrt{2}}+\left(\alpha^{2}-2-\sqrt{2}\right) 2^{n / 2-1}, \\
R_{n}(s) & =s^{2}\left(\frac{1}{1-s}+\frac{1}{2} \sum_{j=1}^{n} \frac{1}{2^{j / 2}-s}\right) .
\end{aligned}
$$

Proof. From (4.22), we see that

$$
2 x p^{2}=\frac{1}{1-s}+\frac{1}{2} \sum_{j=1}^{n} \frac{2^{j}}{\omega^{j}-s}-\frac{1}{2}\left(\frac{2}{\omega}\right)^{n} \alpha^{2} .
$$

Substituting

$$
\frac{2^{j}}{\omega^{j}-s}=\left(\frac{2}{\omega}\right)^{j}+\left(\frac{2}{\omega^{2}}\right)^{j} s+\left(\frac{2}{\omega^{3}}\right)^{j} \frac{s^{2}}{1-\frac{s}{\omega^{j}}}, \quad j \geq 0,
$$

into (4.46), we obtain

$$
2 x p^{2}=-\gamma_{n}+\delta_{n}^{-1} s+R_{n}(s),
$$

where

$$
\begin{aligned}
\gamma_{n} & =\frac{\omega-1}{2-\omega}+\left(\frac{1}{2} \alpha^{2}-\frac{1}{2-\omega}\right)\left(\frac{2}{\omega}\right)^{n}, \\
\delta_{n}^{-1} & =1+\frac{1}{2} \sum_{j=1}^{n}\left(\frac{2}{\omega^{2}}\right)^{j}, \\
R_{n}(s) & =\frac{s^{2}}{1-s}+\frac{1}{2} \sum_{j=1}^{n}\left(\frac{2}{\omega^{3}}\right)^{j} \frac{s^{2}}{1-\omega^{-j} s} .
\end{aligned}
$$

Since $\omega=\sqrt{2}$, we have the lemma.

Remark. Eq.(4.44) implies that the critical value of $\alpha$ is $\sqrt{2+\sqrt{2}}$.

Consider the case $x=0$ in (4.42). Then, $\sigma_{n}=s(0)$ satisfies

$$
\sigma_{n}=\delta_{n}\left(\gamma_{n}-R_{n}\left(\sigma_{n}\right)\right), \quad \sigma_{n}<1 .
$$

In (4.47), if $\delta_{n}$ is small and if $\left|\gamma_{n}\right|$ is not large, then we see that $\sigma_{n}=\mathcal{O}\left(\delta_{n}\right)$. To be precise, using successive approximations, we can show

$$
\left|\sigma_{n}\right|<C_{4} \delta_{n}
$$

for $n$ and $\alpha$ satisfying e.g.

$$
\begin{aligned}
\delta_{n} & <\frac{1}{10} \\
\left|\gamma_{n}\right| & <10
\end{aligned}
$$

where $C_{4}$ is a positive constant. 
Lemma 4.5 Suppose that $n$ and $\alpha$ satisfy (4.50) and

$$
n>C_{5} .
$$

Then, it holds that

$$
\begin{aligned}
C_{6} \delta_{n}<\nu_{4, n}^{(\infty)} & <C_{7} \delta_{n} \\
\left|\nu_{4, n}^{(\infty)}-\delta_{n}\right| & <C_{8} \delta_{n}^{2}, \\
\left|\zeta_{k, n}^{(\infty)}\right| & <C_{9}, \quad k=6,8 .
\end{aligned}
$$

Furthermore, if we write

$$
\zeta_{2, n}^{(\infty)}=\frac{\delta_{n}}{\nu_{4, n}^{(\infty)}}\left(\gamma_{n}-\frac{1}{\sqrt{2}}\right)+Z_{n}
$$

then $Z_{n}$ has the bound

$$
\left|Z_{n}\right|<C_{10}
$$

In the above, $C_{m}$ 's are positive constants independent of $n$.

Proof. We choose the constant $C_{5}$ sufficiently large so that (4.50) and (4.51) imply

$$
\begin{aligned}
& \left|\sigma_{n}\right|<C_{4} \delta_{n}<\frac{1}{2}, \\
& \delta_{n}\left|R_{n}^{\prime}\left(\sigma_{n}\right)\right|<\frac{1}{2} .
\end{aligned}
$$

Now, (4.41) and (4.42) imply that derivatives of $s=s(x)$ and of $p=p(x)$ are given by

$$
\begin{aligned}
& s^{\prime}(x)=2 \delta_{n} p(x)^{2} D_{n}(x), \\
& p^{\prime}(x)=p(x)^{2} s^{\prime}(x)=2 \delta_{n} p(x)^{4} D_{n}(x),
\end{aligned}
$$

respectively, where

$$
D_{n}(x)=\frac{1}{1-4 \delta_{n} x p(x)^{3}+\delta_{n} R_{n}^{\prime}(s(x))} .
$$

Then, it holds that

$$
p^{\prime}(0)=\frac{2 \delta_{n}}{\left(1-\sigma_{n}\right)^{4}\left(1+\delta_{n} R_{n}^{\prime}\left(\sigma_{n}\right)\right)},
$$

from which together with (4.40) we obtain (4.52) and (4.53). Furthermore, from (4.58) we have

$$
p^{\prime \prime}(x)=8 \delta_{n} p(x)^{3} p^{\prime}(x) D_{n}(x)+2 \delta_{n} p(x)^{4} D_{n}^{\prime}(x) .
$$

This implies

$$
p^{\prime \prime}(0)-24 \delta_{n}^{2}=8 \delta_{n}\left(p(0)^{3} p^{\prime}(0) D_{n}(0)-2 \delta_{n}\right)+2 \delta_{n}\left(p(0)^{4} D_{n}^{\prime}(0)-4 \delta_{n}\right)
$$

and hence

$$
\left|p^{\prime \prime}(0)-24 \delta_{n}^{2}\right| \leq \text { const. } \delta_{n}^{3} .
$$

Then, we have the bound (4.54) on $\zeta_{6, n}^{(\infty)}$. Similarly, differentiating the both sides of (4.60), we obtain the bound on $\zeta_{8, n}^{(\infty)}$.

Let us show (4.56). Since (4.47) imply

$$
\begin{aligned}
p(0)-1-\frac{1}{\sqrt{2}} \delta_{n} & =\sigma_{n}+\sigma_{n}^{2} p(0)-\frac{1}{\sqrt{2}} \delta_{n} \\
& =\delta_{n}\left(\gamma_{n}-\frac{1}{\sqrt{2}}\right)-\delta_{n} R_{n}\left(\sigma_{n}\right)+\sigma_{n}^{2} p(0),
\end{aligned}
$$


we have

$$
\nu_{2, n}^{(\infty)}-1-\frac{1}{\sqrt{2}} \nu_{4, n}^{(\infty)}=\delta_{n}\left(\gamma_{n}-\frac{1}{\sqrt{2}}\right)-\delta_{n} R_{n}\left(\sigma_{n}\right)+\sigma_{n}^{2} p(0)+\frac{1}{\sqrt{2}}\left(\delta_{n}-\nu_{4, n}^{(\infty)}\right)
$$

This yields

$$
Z_{n}=\frac{1}{\nu_{4, n}^{(\infty)^{2}}}\left(-\delta_{n} R_{n}\left(\sigma_{n}\right)+\sigma_{n}^{2} p(0)+\frac{1}{\sqrt{2}}\left(\delta_{n}-\nu_{4, n}^{(\infty)}\right)\right)
$$

and hence the bound 4.56).

Proof of Proposition 2.2. We choose $n_{1}$ sufficiently large so that (4.51) and

$$
\delta_{n_{1}}<\min \left(\frac{1}{C_{7}^{2}\left(2 \zeta+C_{10}\right)}, \frac{\epsilon}{2 C_{7}}, \frac{\epsilon_{0}}{2 C_{7} C_{9}}, \frac{\epsilon_{1}}{2 C_{7} C_{9}},\right)
$$

hold. By virtue of (4.44), there exist $\alpha_{++}$and $\alpha_{--}$such that

$$
\begin{aligned}
& \gamma_{n_{1}}-\frac{1}{\sqrt{2}}=C_{7}^{2}\left(2 \zeta+C_{10}\right) \delta_{n_{1}}, \quad \text { at } \alpha=\alpha_{++}, \\
& \gamma_{n_{1}}-\frac{1}{\sqrt{2}}=-C_{7}^{2}\left(2 \zeta+C_{10}\right) \delta_{n_{1}}, \quad \text { at } \alpha=\alpha_{--} .
\end{aligned}
$$

Since

$$
\left|\gamma_{n_{1}}-\frac{1}{\sqrt{2}}\right| \leq 1, \quad \alpha \in\left[\alpha_{--}, \alpha_{++}\right],
$$

(4.50) holds for $n=n_{1}$ and $\alpha \in\left[\alpha_{--}, \alpha_{++}\right]$. Then, (4.52) and (4.54) imply (2.34)-(2.36). Furthermore, (4.55) and (4.56) imply (2.32) and (2.33).

\section{From $\mathcal{O}(N)$ trajectory to $\mathcal{O}(\infty)$ trajectory}

In Section 4 we obtained the solution $\nu_{k, n}^{(\infty)}(t)$ to the system of ordinary differential equations (4.36)(4.38) with (4.39). This system is the formal limit of (2.13) 2.15) as $N \rightarrow \infty$. In this section, we show Proposition 2.3. namely, the fact that the solution $\nu_{k, n}^{(N)}(t)$ to 2.13) 2.15) is convergent as $N \rightarrow \infty$ and the limit coincides with $\nu_{k, n}^{(\infty)}(t)$.

This fact is by no means trivial, because (2.10) is a singular perturbation of (2.24) and because the initial value problem for the infinite dimensional system of ordinary differential equations lacks uniqueness of solution. In order to show that $\nu_{k, n}^{(N)}(t)$ is convergent, we use $1 / N$ expansion developed by Kupiainen 9]. His method for spin systems on regular lattices also applies to our hierarchical model.

\subsection{Boundedness}

We begin with the basic bound on the 'mass term' $\nu_{2, n}^{(N)}(t)$ uniform in $N$.

Lemma 5.1 For $t \geq 0, n \geq 1, \alpha>0$ and $N \geq 1$, it holds that

$$
0 \leq \nu_{2, n}^{(N)}(t) \leq \frac{1}{2} \alpha^{2}\left(\frac{2}{\omega}\right)^{n}
$$


Proof. We go back to the renormalization group transformation (1.8) in the configuration space:

$$
h_{n}^{(N)}(t, \boldsymbol{x})=\frac{1}{Z_{n}^{(N)}(t)} e^{t|\boldsymbol{x}|^{2}} \int_{\mathbb{R}^{N}} d \boldsymbol{y} h_{n-1}^{(N)}\left(\sqrt{\frac{\omega}{2}} \boldsymbol{x}+\boldsymbol{y}\right) h_{n-1}^{(N)}\left(\sqrt{\frac{\omega}{2}} \boldsymbol{x}-\boldsymbol{y}\right),
$$

where

$$
Z_{n}^{(N)}(t)=\int_{\mathbb{R}^{N}} d \boldsymbol{x} e^{t|\boldsymbol{x}|^{2}} \int_{\mathbb{R}^{N}} d \boldsymbol{y} h_{n-1}^{(N)}\left(\sqrt{\frac{\omega}{2}} \boldsymbol{x}+\boldsymbol{y}\right) h_{n-1}^{(N)}\left(\sqrt{\frac{\omega}{2}} \boldsymbol{x}-\boldsymbol{y}\right) .
$$

Note that $\nu_{2, n}^{(N)}(t)$ is related to the moment of the measure $h_{n}^{(N)}(t, \boldsymbol{x}) d \boldsymbol{x}$ as

$$
\nu_{2, n}^{(N)}(t)=\frac{1}{2} \int_{\mathbb{R}^{N}} x_{1}^{2} h_{n}^{(N)}(t, \boldsymbol{x}) d \boldsymbol{x}=\frac{1}{2 N} \int_{\mathbb{R}^{N}}|\boldsymbol{x}|^{2} h_{n}^{(N)}(t, \boldsymbol{x}) d \boldsymbol{x},
$$

where $\boldsymbol{x}=\left(x_{1}, x_{2}, \cdots, x_{N}\right)$.

Let $r_{n}$ be the radius of $\operatorname{Supp} h_{n}^{(N)}(\cdot)$ for $n \geq 0$. Since $\operatorname{Supp} h_{n}^{(N)}(t, \cdot)=\operatorname{Supp} h_{n}^{(N)}(\cdot)$ for $t \geq 0$, we see that $r_{n}$ 's satisfy

$$
r_{n} \leq \sqrt{\frac{2}{\omega}} r_{n-1}, n \geq 1 .
$$

Hence we have

$$
r_{n} \leq r_{0}\left(\frac{2}{\omega}\right)^{n / 2}=\sqrt{N} \alpha\left(\frac{2}{\omega}\right)^{n / 2},
$$

which together with (5.4) implies (5.1).

Based on the above lemma, we show the following bound independent of $N$.

Lemma 5.2 For $j \geq 1, n \geq 1$ and $\alpha_{2}>\alpha_{1}>0$, there exists a positive constant $C_{11}=C_{11}\left(2 j, n, \alpha_{1}, \alpha_{2}\right)$ such that

$$
0 \leq \nu_{2 j, n}^{(N)}(t) \leq C_{11}
$$

holds for $\alpha \in\left[\alpha_{1}, \alpha_{2}\right], t \in[0, \beta / 2]$ and $N \geq 1$.

Proof. For $n=0$, the lemma follows from Lemma 4.1

Let $n \geq 1$. If $j=1$, (5.1) implies (5.5). Let $j>1$. We drop the last term in the right hand side of (2.13) so that we obtain

$$
\nu_{2 j, n}^{(N)}(t) \leq \frac{2}{(2 \omega)^{j}} \nu_{2 j, n-1}^{(N)}+\int_{0}^{t} \sum_{\substack{m+\ell=2 j+2 \\ m, \ell \geq 2}} m \ell \nu_{m, n}^{(N)}(\tau) \nu_{\ell, n}^{(N)}(\tau) d \tau .
$$

Then, we can show the lemma by induction on $n$ and $j$.

\subsection{Convergence}

We now employ the $1 / N$ expansion. Let us consider the $\phi$ representation (1.1)-(1.4) in the following form:

$$
\begin{aligned}
\phi_{\theta} & =\phi_{\theta_{\Lambda}, \ldots, \theta_{1}}, \quad \theta=\left(\theta_{\Lambda}, \ldots, \theta_{1}\right) \in \mathcal{L}_{\Lambda} \\
\langle F\rangle_{\Lambda, t} & =\frac{1}{Z_{\Lambda}^{(N)}(t)} \int d \phi F(\phi) \exp \left(\frac{1}{2}\left(\phi, J_{\Lambda}(t) \phi\right)\right) \prod_{\theta \in \mathcal{L}_{\Lambda}} \delta\left(\left|\phi_{\theta}\right|-\sqrt{N} \alpha\right), \\
\left(\phi, J_{\Lambda}(t) \phi\right) & =\frac{2 t}{(2 \omega)^{\Lambda}}\left|\sum_{\theta_{\Lambda}, \ldots, \theta_{1}=0,1} \phi_{\theta_{\Lambda}, \ldots, \theta_{1}}\right|^{2}+\sum_{k=1}^{\Lambda-1} \frac{\beta}{(2 \omega)^{k}} \sum_{\theta_{\Lambda}, \ldots, \theta_{k+1}=0,1}\left|\sum_{\theta_{k}, \ldots, \theta_{1}=0,1} \phi_{\theta_{\Lambda}, \ldots, \theta_{1}}\right|^{2}, \\
Z_{\Lambda}^{(N)}(t) & =\int d \phi \exp \left(\frac{1}{2}\left(\phi, J_{\Lambda}(t) \phi\right)\right) \prod_{\theta \in \mathcal{L}_{\Lambda}} \delta\left(\left|\phi_{\theta}\right|-\sqrt{N} \alpha\right) .
\end{aligned}
$$


Note that we have introduced $t$-dependence in the right hand side of (5.8) in order to study the $t$-dependent correlation $\nu_{k, n}^{(N)}(t)$.

Let $\phi_{\theta}^{(i)}, i=1,2, \cdots, N$, be the $i$-th component of $\phi_{\theta} \in \mathbb{R}^{N}$. For a set $A$ of lattice points in $\mathcal{L}_{\Lambda}$, same point possibly occurring several times, we write

$$
\phi_{A}^{(i)}=\prod_{\theta \in A} \phi_{\theta}^{(i)} .
$$

Let

$$
\left\langle\prod_{i=1}^{N} \phi_{A_{i}}^{(i)}\right\rangle_{\Lambda, t}=\sum_{m=0}^{\infty} s_{m}\left(\Lambda, t, \alpha,\left\{A_{i}\right\}\right) N^{-m}
$$

be the formal $1 / N$ expansion of the correlation $\left\langle\prod_{i=1}^{N} \phi_{A_{i}}^{(i)}\right\rangle_{\Lambda, t}$. (See [9 P.278].)

Proposition 5.3 9] Theorem 2] For $r>0, \alpha_{2}>\alpha_{1}>0, \Lambda \geq 2$, there exists a positive constant $C_{12}=C_{12}\left(r, \alpha_{1}, \alpha_{2}, \Lambda\right)$ such that, for $t \in[0, \beta / 2], \alpha \in\left(\alpha_{1}, \alpha_{2}\right)$ and $N>C_{12}$, the remainder of the $1 / N$ expansion up to $\mathcal{O}\left(N^{-r+1}\right)$ has the bound

$$
\left|\left\langle\prod_{i=1}^{N} \phi_{A_{i}}^{(i)}\right\rangle_{\Lambda, t}-\sum_{m=0}^{r-1} s_{m}\left(\Lambda, t, \alpha,\left\{A_{i}\right\}\right) N^{-m}\right| \leq R\left(r, \alpha_{1}, \alpha_{2}, \Lambda,\left\{A_{i}\right\}\right) N^{-r},
$$

where $R\left(r, \alpha_{1}, \alpha_{2}, \Lambda,\left\{A_{i}\right\}\right)$ is a constant independent of $N$.

Remark. The above proposition can be proved along the same line of argument as 9. In Appendix $\mathrm{A}$ we check the properties to be assumed for applying the method of 9 to our hierarchical system (5.6) (5.9). Note also that $\Lambda \geq 2$ should be assumed, since we need bounds uniform in $t \in[0, \beta / 2]$. $\left(J_{1}(t)\right.$ vanishes as $t \rightarrow 0$.)

Proposition 5.3 plays a key role in the proof of the lemma below (for $n \geq 2$ ).

Lemma 5.4 For $j \geq 1$ and $n \geq 1$, the limit

$$
\bar{\nu}_{2 j, n}(t)=\lim _{N \rightarrow \infty} \nu_{2 j, n}^{(N)}(t)
$$

exists, where the convergence is uniform in $t \in[0, \beta / 2]$ and in $\alpha$ on any compact subset of $(0, \infty)$.

Remark. In the proof below, we assume $n \geq 2$ and apply Proposition 5.3 The case $n=1$ is dealt with in Section 6

Proof. Let $n \geq 2$. Put, for $j \geq 1$,

$$
\begin{aligned}
a_{2 j, n}^{(N)}(t) & =\left\langle x_{1}^{2 j}\right\rangle_{n, t}, \\
a_{2 j, n(c)}^{(N)}(t) & =(-1)^{j-1} \frac{(2 j) !}{N^{j-1}} \nu_{2 j, n}^{(N)}(t),
\end{aligned}
$$

where $x_{1}$ denotes the first component of the block spin

$$
\boldsymbol{x}=\frac{1}{(2 \omega)^{n / 2}} \sum_{\theta_{n}, \ldots, \theta_{1}} \phi_{\theta_{n}, \ldots, \theta_{1}} .
$$

Note that $a_{2 j, n(c)}^{(N)}(t)$ is the connected part of $a_{2 j, n}^{(N)}(t)$, namely,

$$
a_{2 j, n(c)}^{(N)}(t)=\left\langle x_{1} ; x_{1} ; \cdots ; x_{1}\right\rangle_{n, t} .
$$


Applying Proposition 5.3 we expand correlations $a_{2 j, n}^{(N)}(t)$ up to $\mathcal{O}\left(N^{-j+1}\right)$ with remainder estimates. These expansions yield an expression for the connected part $a_{2 j, n(c)}^{(N)}(t)$, in which all the terms up to $\mathcal{O}\left(N^{-j+1}\right)$ are explicitly written:

$$
a_{2 j, n(c)}^{(N)}(t)=\sum_{m=0}^{j-1} a_{2 j, n, m(c)}(t) N^{-m}+a_{2 j, n(c)}^{(N) \geq j}(t),
$$

where $a_{2 j, n, m(c)}(t) N^{-m}$ stands for the sum of explicit terms of $\mathcal{O}\left(N^{-m}\right)$ and $a_{2 j, n(c)}^{(N) \geq j}(t)$ denotes the remainder with the property:

$$
\lim _{N \rightarrow \infty} N^{j-1} a_{2 j, n(c)}^{(N) \geq j}(t)=0 .
$$

Since (5.5) implies that the right hand side of (5.14) is uniformly bounded in $N$, we see

$$
a_{2 j, n, m(c)}(t)=0, \quad m=0,1,2, \cdots, j-2,
$$

so that we have

$$
a_{2 j, n(c)}^{(N)}(t)=a_{2 j, n, j-1(c)}(t) N^{-(j-1)}+a_{2 j, n(c)}^{(N) \geq j}(t) .
$$

This implies

$$
\begin{gathered}
\nu_{2 j, n}^{(N)}(t)=\frac{(-1)^{j-1}}{(2 j) !} a_{2 j, n, j-1(c)}(t)+\frac{(-N)^{j-1}}{(2 j) !} a_{2 j, n(c)}^{(N) \geq j}(t) \\
\rightarrow \frac{(-1)^{j-1}}{(2 j) !} a_{2 j, n, j-1(c)}(t), \quad N \rightarrow \infty .
\end{gathered}
$$

\section{$5.3 \quad$ Limit}

Thanks to Lemma 5.4 we can take limits in both sides of (2.13)

$$
\begin{aligned}
\frac{d}{d t} \bar{\nu}_{2 j, n}(t) & =\sum_{\substack{m+\ell=2 j \\
m, \ell \geq 2}} m \ell \bar{\nu}_{m, n}(t) \bar{\nu}_{\ell, n}(t)-(2 j+2) \bar{\nu}_{2 j+2, n}(t), \\
\bar{\nu}_{2 j, n}(0) & =\frac{2}{(2 \omega)^{j}} \bar{\nu}_{2 j, n-1}, \\
\bar{\nu}_{2 j, n} & =\bar{\nu}_{2 j, n}\left(\frac{\beta}{2}\right)
\end{aligned}
$$

and we can write (4.39) as

$$
\bar{\nu}_{2 j, 0}=\nu_{2 j, 0}^{(\infty)} .
$$

Although the system (5.20)-(5.22) has the same form as 4.36)-4.38), we need an additional condition in order to ensure the equality $\bar{\nu}_{2 j, n}(t)=\nu_{2 j, n}^{(\infty)}(t)$.

Lemma 5.5 In addition to (4.36) (4.38) and (5.20)-(5.23), we assume

$$
\bar{\nu}_{2, n}(t)=\nu_{2, n}^{(\infty)}(t), \quad t \in\left[0, \frac{\beta}{2}\right], n \geq 1 .
$$

Then, it holds that

$$
\bar{\nu}_{2 j, n}(t)=\nu_{2 j, n}^{(\infty)}(t), \quad t \in\left[0, \frac{\beta}{2}\right], n \geq 1, j \geq 1 .
$$


Proof. Solving (4.36) and (5.20) with respect to $\nu_{2 j+2, n}^{(\infty)}(t)$ and $\bar{\nu}_{2 j+2, n}(t)$, respectively, we see by induction that (5.25) holds.

Proof of Proposition 2.3. Let us show (5.24). Since $p=u_{n}^{\prime}(t, x)$ is an inverse of $x=w_{n}(t, x)$, (4.10) and (4.35) imply that

$$
\nu_{2, n}^{(\infty)}(t)=u_{n}^{\prime}(t, 0)=\pi_{n}(t)
$$

where $\pi_{n}(t)$ is the unique positive solution to $w_{n}(t, p)=0$ (see Lemma 4.2). Then, in view of (4.23), we see that $\nu_{2, n}^{(\infty)}(t)$ is the unique positive solution to

$$
\alpha^{2}=2\left(\frac{\omega}{2}\right)^{n} \nu_{2, n}^{(\infty)}(t)+\left(\frac{\omega}{2}\right)^{n} \sum_{j=1}^{n} \frac{2^{j}}{\omega^{j}-\omega+4 t+\frac{1}{\nu_{2, n}^{(\infty)}(t)}} .
$$

On the other hand, using the variable $x_{1}$, the first component of $\boldsymbol{x}$ defined by (5.15), we have

$$
\begin{aligned}
\bar{\nu}_{2, n}(t) & =\lim _{N \rightarrow \infty} \nu_{2, n}^{(N)}(t)=\lim _{N \rightarrow \infty} \frac{1}{2}\left\langle x_{1}^{2}\right\rangle_{n, t}, \\
& =\lim _{N \rightarrow \infty} \frac{1}{2(2 \omega)^{n}} \sum_{\theta, \theta^{\prime} \in \mathcal{L}_{n}}\left\langle\phi_{\theta}^{(1)} \phi_{\theta^{\prime}}^{(1)}\right\rangle_{n, t} .
\end{aligned}
$$

In order to calculate $\lim _{N \rightarrow \infty}\left\langle\phi_{\theta}^{(1)} \phi_{\theta^{\prime}}^{(1)}\right\rangle_{n, t}$, we use again the $1 / N$ expansion. (We can assume $n \geq 2$ or $t>0$, since, if $n=1$ and $t=0$, (5.24) has been established in (5.23).) Decompose $J_{n}(t)$ defined by (5.8) with $\Lambda=n$ as follows:

$$
J_{n}(t)=\triangle+\mu_{0} I
$$

Here $I$ denotes the identity matrix and the real constant $\mu_{0}$ is chosen so that

$$
\triangle \mathbf{1}_{n}=\mathbf{0}
$$

holds, where we denoted $\mathbf{1}_{n}={ }^{t}(1,1, \ldots, 1) \in \mathbb{R}^{\mathcal{L}_{n}}$.

Now, put

$$
C=\left(-\triangle+m^{2} I\right)^{-1}
$$

for $m^{2}>0$.

\section{Lemma 5.6 It holds that}

$$
\begin{aligned}
\sum_{\theta, \theta^{\prime} \in \mathcal{L}_{n}} C_{\theta \theta^{\prime}} & =\frac{2^{n}}{m^{2}}, \\
C_{\theta \theta} & =\left(\frac{\omega}{2}\right)^{n} \sum_{j=1}^{n} \frac{2^{j}}{\omega^{j}-\omega+4 t+2 \omega^{n} m^{2}}+\frac{1}{2^{n} m^{2}}, \quad \theta \in \mathcal{L}_{n} .
\end{aligned}
$$

This lemma will be shown in Appendix A.2

Now, choose $m^{2}$ so that $C_{\theta \theta}=\alpha^{2}$ holds, i.e.,

$$
\left(\frac{\omega}{2}\right)^{n} \sum_{j=1}^{n} \frac{2^{j}}{\omega^{j}-\omega+4 t+2 \omega^{n} m^{2}}+\frac{1}{2^{n} m^{2}}=\alpha^{2} .
$$

Then, we have [9, P.284]

$$
\lim _{N \rightarrow \infty}\left\langle\phi_{\theta}^{(1)} \phi_{\theta^{\prime}}^{(1)}\right\rangle_{n, t}=C_{\theta \theta^{\prime}}
$$

Now, (5.33) together with (5.28) and (5.30) implies

$$
\bar{\nu}_{2, n}(t)=\frac{1}{2} \frac{1}{(2 \omega)^{n}} \sum_{\theta, \theta^{\prime} \in \mathcal{L}_{n}} C_{\theta \theta^{\prime}}=\frac{1}{2 \omega^{n} m^{2}} .
$$

Thus, we obtain (5.24) from (5.32), (5.34) and (5.27).

Since (5.24) holds, Lemma 5.4 and Lemma 5.5 yield Proposition 2.3 


\section{The first effective theory}

In this section, we prove Lemma 5.4 for $n=1$. For this purpose, we study the first effective measure density:

$$
h_{1}^{(N)}(t, \boldsymbol{x})=\frac{1}{Z_{1}^{(N)}(t)} \exp \left(t|\boldsymbol{x}|^{2}\right) \int_{\mathbb{R}^{N}} h_{0}^{(N)}\left(\sqrt{\frac{\omega}{2}} \boldsymbol{x}+\boldsymbol{y}\right) h_{0}^{(N)}\left(\sqrt{\frac{\omega}{2}} \boldsymbol{x}-\boldsymbol{y}\right) d \boldsymbol{y}, \quad \boldsymbol{x} \in \mathbb{R}^{N},
$$

where

$$
Z_{1}^{(N)}(t)=\int_{\mathbb{R}^{N}} d \boldsymbol{x} \exp \left(t|\boldsymbol{x}|^{2}\right) \int_{\mathbb{R}^{N}} d \boldsymbol{y} h_{0}^{(N)}\left(\sqrt{\frac{\omega}{2}} \boldsymbol{x}+\boldsymbol{y}\right) h_{0}^{(N)}\left(\sqrt{\frac{\omega}{2}} \boldsymbol{x}-\boldsymbol{y}\right)
$$

Let us denote integrations with respect to the measures $h_{0}^{(N)}(\boldsymbol{x}) d \boldsymbol{x}$ and $h_{1}^{(N)}(t, \boldsymbol{x}) d \boldsymbol{x}$ by $\langle\cdot\rangle_{0}$ and $\langle\cdot\rangle_{1}$, respectively.

\section{Lemma 6.1 Put}

$$
A_{m}=\prod_{k=0}^{m-1}\left(1+\frac{2 k}{N}\right)^{-1}, \quad m \geq 1
$$

Then, we have

$$
\begin{aligned}
\hat{h}_{0}^{(N)}(\xi) & =1+\sum_{m=1}^{\infty} \frac{\left(-\alpha^{2}\right)^{m}}{2^{m} m !} A_{m} \xi^{2 m} \\
\hat{h}_{1}^{(N)}(t, \xi) & =1+\sum_{m=1}^{\infty} \frac{(-1)^{m}}{(2 N)^{m} m !} A_{m}\left\langle|x|^{2 m}\right\rangle_{1} \xi^{2 m} .
\end{aligned}
$$

Proof. The equality (6.3) follows from (4.2). Let us show 6.4).

By virtue of spherical symmetry, we have

$$
\hat{h}_{1}^{(N)}(t, \xi)=\left\langle e^{\sqrt{-1}(\boldsymbol{\xi}, \boldsymbol{x})}\right\rangle_{1}=\left\langle e^{\sqrt{-1} \xi x_{1}}\right\rangle_{1}=\sum_{m=0}^{\infty} \frac{\left(-\xi^{2}\right)^{m}}{(2 m) !}\left\langle x_{1}^{2 m}\right\rangle_{1},
$$

where $\xi=|\boldsymbol{\xi}|$ and $x_{1}$ is the first component of $\boldsymbol{x} \in \mathbb{R}^{N}$. Similarly, we have

$$
\hat{h}_{0}^{(N)}(\xi)=\sum_{m=0}^{\infty} \frac{\left(-\xi^{2}\right)^{m}}{(2 m) !}\left\langle x_{1}^{2 m}\right\rangle_{0}
$$

Comparing (6.6) with (6.3), we see that

$$
\left\langle x_{1}^{2 m}\right\rangle_{0}=\frac{\alpha^{2 m}(2 m) !}{2^{m} m !} A_{m}=\frac{(2 m) !}{(2 N)^{m} m !} A_{m}\left\langle|\boldsymbol{x}|^{2 m}\right\rangle_{0} .
$$

Now, note that the expectation $\langle\cdot\rangle_{0}$ gives average over an $N$ dimensional sphere. Then, integrating with respect to the radial distribution of $\hat{h}_{1}^{(N)}(t, \boldsymbol{\xi})$, we obtain from (6.7)

$$
\left\langle x_{1}^{2 m}\right\rangle_{1}=\frac{(2 m) !}{(2 N)^{m} m !} A_{m}\left\langle|\boldsymbol{x}|^{2 m}\right\rangle_{1}
$$

This together with (6.5) gives (6.4).

Now let us introduce the connected part

$$
\left\langle|\boldsymbol{x}|^{2 k}\right\rangle_{1, c c}=\left\langle|\boldsymbol{x}|^{2} ;|\boldsymbol{x}|^{2} ; \cdots ;|\boldsymbol{x}|^{2}\right\rangle_{1}
$$

of $\left\langle|\boldsymbol{x}|^{2 k}\right\rangle_{1}$ with $|\boldsymbol{x}|^{2}$ as units, namely, we define $\left\langle|\boldsymbol{x}|^{2 k}\right\rangle_{1, c c}, k=1,2, \cdots$, by

$$
\sum_{m=0}^{\infty} \frac{\left\langle|\boldsymbol{x}|^{2 m}\right\rangle_{1}}{m !} z^{m}=\exp \left(\sum_{k=1}^{\infty} \frac{\left\langle|\boldsymbol{x}|^{2 k}\right\rangle_{1, c c}}{k !} z^{k}\right)
$$


or, equivalently

$$
\left\langle|\boldsymbol{x}|^{2 m}\right\rangle_{1}=\sum_{n \geq 1} \sum_{\sum_{j=1}^{n} k_{j}=m} \frac{1}{n !} \frac{m !}{\prod_{j=1}^{n} k_{j} !} \prod_{j=1}^{n}\left\langle|\boldsymbol{x}|^{2 k_{j}}\right\rangle_{1, c c}, \quad m \geq 1
$$

where the summations are taken over the sets of all $\left(k_{1}, k_{1}, \cdots, k_{n}\right) \in \mathbb{N}^{n}$ with $\sum_{j=1}^{n} k_{j}=m$ and over $n \in \mathbb{N}$. Note that we can get information on the connected part $\left\langle|\boldsymbol{x}|^{2 k}\right\rangle_{1, c c}$ from $Z_{1}^{(N)}(t)$, since

$$
\left\langle|\boldsymbol{x}|^{2 k}\right\rangle_{1, c c}=\left(\frac{d}{d t}\right)^{k} \log Z_{1}^{(N)}(t), \quad k \geq 1
$$

Lemma 6.2 For $k=1,2, \cdots$, the limit $\lim _{N \rightarrow \infty} \frac{1}{N}\left\langle|\boldsymbol{x}|^{2 k}\right\rangle_{1, c c}$ exists, where the convergence is uniform in $t \in[0, \beta / 2]$ and in $\alpha$ on any compact subset of $(0, \infty)$.

Proof. We carry out the integrations in the right hand side of (6.1):

$$
\begin{aligned}
Z_{1}^{(N)}(t) & =\text { const. } \int_{\mathbb{R}^{N}} d \boldsymbol{x} \int_{\mathbb{R}^{N}} d \boldsymbol{y} \exp \left(\frac{t}{2 \omega}|\boldsymbol{x}+\boldsymbol{y}|^{2}\right) h_{0}^{(N)}(\boldsymbol{x}) h_{0}^{(N)}(\boldsymbol{y}) \\
& =\text { const. } \exp \left(\frac{t}{\omega} N \alpha^{2}\right) \int_{\mathbb{R}^{N}} d \boldsymbol{x} \int_{\mathbb{R}^{N}} d \boldsymbol{y} \exp \left(\frac{t}{\omega}(\boldsymbol{x}, \boldsymbol{y})\right) \delta(|\boldsymbol{x}|-\sqrt{N} \alpha) \delta(|\boldsymbol{y}|-\sqrt{N} \alpha) \\
& =\text { const. } \exp \left(\frac{t}{\omega} N \alpha^{2}\right) \int_{\mathbb{R}^{N}} d \boldsymbol{x} \delta(|\boldsymbol{x}|-\sqrt{N} \alpha) \hat{h}_{0}^{(N)}\left(\frac{t}{\sqrt{-1} \omega}|\boldsymbol{x}|\right) \\
& =\text { const. } \exp \left(\frac{t}{\omega} N \alpha^{2}\right) \hat{h}_{0}^{(N)}\left(\frac{t}{\sqrt{-1} \omega} \sqrt{N} \alpha\right) .
\end{aligned}
$$

Therefore, we have

$$
\frac{1}{N} \log Z_{1}^{(N)}(t)=\text { const. }+\frac{t}{\omega} \alpha^{2}-v_{0}^{(N)}\left(\frac{t \alpha}{\sqrt{-1} \omega}\right)
$$

Lemma 4.1 implies that the right hand side of (6.12) converges as $N \rightarrow \infty$, where the convergence is uniform in $t$ on any compact subset of a certain neighborhood of the real axis and in $\alpha$ on any compact subset of $(0, \infty)$. Then, the limit

$$
\lim _{N \rightarrow \infty} \frac{1}{N}\left\langle|\boldsymbol{x}|^{2 k}\right\rangle_{1, c c}=\lim _{N \rightarrow \infty} \frac{1}{N}\left(\frac{d}{d t}\right)^{k} \log Z_{1}^{(N)}(t)
$$

exists for $k \geq 1, t \in[0, \beta / 2]$ and for $\alpha>0$. The convergence is uniform in $t \in[0, \beta / 2]$ and in $\alpha$ on any compact subset of $(0, \infty)$.

Next, we define the connected part $A_{k, c}$ of $A_{k}$ by

$$
1+\sum_{m=1}^{\infty} \frac{A_{m}}{m !} z^{m}=\exp \left(\sum_{k=1}^{\infty} \frac{A_{k, c}}{k !} z^{k}\right)
$$

or equivalently

$$
A_{m}=\sum_{n \geq 1} \sum_{\sum_{j=1}^{n} k_{j}=m} \frac{1}{n !} \frac{m !}{\prod_{j=1}^{n} k_{j} !} \prod_{j=1}^{n} A_{k_{j}, c}, \quad m \geq 1 .
$$

Lemma 6.3 For $k=1,2, \cdots$, the limit $\lim _{N \rightarrow \infty} N^{k-1} A_{k, c}$ exists. 
Proof. From (6.3) and 6.13), we have

$$
\hat{h}_{0}^{(N)}(\xi)=\exp \left(\sum_{k=1}^{\infty} \frac{A_{k, c}}{k !}\left(-\frac{1}{2} \alpha^{2} \xi^{2}\right)^{k}\right)
$$

and hence

$$
v_{0}^{(N)}(\eta)=-\frac{1}{N} \sum_{k=1}^{\infty} \frac{A_{k, c}}{k !}\left(-\frac{1}{2} \alpha^{2} N \eta^{2}\right)^{k}
$$

Then, it holds that

$$
N^{k-1} A_{k, c}=(-1)^{k-1} \frac{k ! 2^{k}}{\alpha^{2 k}} \nu_{2 k, 0}^{(N)} .
$$

Since Lemma 4.1 implies that $\nu_{2 k, 0}^{(N)}$ converges as $N \rightarrow \infty$, we obtain the lemma.

Put, for $m \geq 1$,

$$
\begin{aligned}
B_{m} & =\left\langle|\boldsymbol{x}|^{2 m}\right\rangle_{1}, \\
B_{m, c} & =\left\langle|\boldsymbol{x}|^{2 m}\right\rangle_{1, c c},
\end{aligned}
$$

and write (6.4) as

$$
\hat{h}_{1}^{(N)}(\xi)=\sum_{m=1}^{\infty} \frac{A_{m} B_{m}}{m !}\left(-\frac{\xi^{2}}{2 N}\right)^{m} .
$$

We now introduce connected parts of the product " $A B$ ". Let $\mathcal{P}_{m}$ be the set of all partitions of $\{1,2, \cdots, m\}$. Then, (6.14) and (6.10) can be written as

$$
\begin{aligned}
& A_{m}=\sum_{P \in \mathcal{P}_{m}} \prod_{I \in P} A_{|I|, c}, \\
& B_{m}=\sum_{P \in \mathcal{P}_{m}} \prod_{I \in P} B_{|I|, c},
\end{aligned}
$$

respectively, where $|I|$ denotes the number of elements of $I$. Here, we think each $I \in \mathcal{P}_{m}$ to connect elements contained in $I$ into a single component by $|I|-1$ links (bonds). The connected part of " $A B$ " is by definition

$$
(A B)_{k, c}=\sum_{P, Q \in \mathcal{P}_{k}} \chi_{k}(P, Q) \prod_{I \in P} A_{|I|, c} \prod_{J \in Q} B_{|J|, c}
$$

where

$$
\chi_{k}(P, Q)= \begin{cases}1, & \{1,2, \cdots, k\} \text { is connected by } P \cup Q, \\ 0, & \text { otherwise } .\end{cases}
$$

In this notation, we can write [6.15] as

$$
\hat{h}_{1}^{(N)}(\xi)=\exp \left(\sum_{k=1}^{\infty} \frac{(A B)_{k, c}}{k !}\left(-\frac{\xi^{2}}{2 N}\right)^{k}\right) .
$$

Thus, we obtain the formula:

$$
\nu_{2 k, 1}^{(N)}(t)=-\frac{1}{N}\left(-\frac{1}{2}\right)^{k} \frac{(A B)_{k, c}}{k !}
$$

Lemma 6.4 For $k=1,2,3, \cdots$, the limit $\lim _{N \rightarrow \infty} \frac{1}{N}(A B)_{k, c}$ exists, where the convergence is uniform in $t \in[0, \beta / 2]$ and in $\alpha$ on any compact subset of $(0, \infty)$. 
Proof. Consider the term

$$
\psi(P, Q)=\prod_{I \in P} A_{|I|, c} \prod_{J \in Q} B_{|J|, c}
$$

in the right hand side of (6.16) corresponding to a pair of partitions $P, Q \in \mathcal{P}_{k}$ with $\chi_{k}(P, Q)=1$. Suppose that the partition $P$ consists of $n$ elements, that is, the set $\{1,2, \cdots, k\}$ is decomposed into $n$ disjoint subsets each of which is connected by some element of $P$. Then, Lemma 6.3 implies that the quantity

$$
N^{k-n} \prod_{I \in P} A_{|I|, c}=\prod_{I \in P} N^{|I|-1} A_{|I|, c}
$$

is convergent as $N \rightarrow \infty$. On the other hand, $Q$ should have at least $n-1$ links because otherwise the set $\{1,2, \cdots, k\}$ could not be connected by $P \cup Q$. Let us denote the number of elements of $Q$ by $n^{\prime}$. Then, we have $n^{\prime} \leq k-n+1$ and Lemma 6.2 implies that

$$
N^{-k+n-1} \prod_{J \in Q} B_{|J|, c}=N^{n^{\prime}-(k-n+1)} \prod_{J \in Q} N^{-1} B_{|J|, c}
$$

is convergent. Thus, we see that

$$
\frac{1}{N} \psi(P, Q)=N^{k-n} \prod_{I \in P} A_{|I|, c} \cdot N^{-k+n-1} \prod_{J \in Q} B_{|J|, c}
$$

is convergent and we obtain the lemma.

Lemma 5.4 for $n=1$ directly follows from Lemma 6.4 and (6.18).

Acknowledgments. I would like to thank T.Hattori and T.Hara for valuable discussions and comments.

\section{A Hierarchical Gaussian}

In this appendix, we show the properties to be assumed when we apply the $1 / N$ expansion 9 to the proof of Proposition 5.3 we prove the reflection positivity (Lemma A.1) and certain bounds on the hierarchical Laplacian (Lemma A.2). In addition we prove Lemma 5.6

Let us write (5.6) (5.9) as follows:

$$
\begin{aligned}
\phi_{\theta} & =\phi_{\theta_{\Lambda}, \ldots, \theta_{1}}, \quad \theta=\left(\theta_{\Lambda}, \ldots, \theta_{1}\right) \in \mathcal{L}_{\Lambda}, \\
\langle F\rangle_{J} & =\frac{1}{Z_{J}} \int d \phi F(\phi) \exp \left(\frac{1}{2}(\phi, J \phi)\right) \prod_{\theta \in \mathcal{L}_{\Lambda}} \delta\left(\left|\phi_{\theta}\right|^{2}-N \alpha^{2}\right), \\
(\phi, J \phi) & =\sum_{k=1}^{\Lambda} \frac{\beta_{k}}{(2 \omega)^{k}} \sum_{\theta_{\Lambda}, \ldots, \theta_{k+1}=0,1}\left|\sum_{\theta_{k}, \ldots, \theta_{1}=0,1} \phi_{\theta_{\Lambda}, \ldots, \theta_{1}}\right|^{2}, \\
Z_{J}(t) & =\int d \phi \exp \left(\frac{1}{2}(\phi, J \phi)\right) \prod_{\theta \in \mathcal{L}_{\Lambda}} \delta\left(\left|\phi_{\theta}\right|^{2}-N \alpha^{2}\right),
\end{aligned}
$$

where

$$
\beta_{k}= \begin{cases}\beta, & k=1,2, \cdots, \Lambda-1 \\ 2 t, & k=\Lambda\end{cases}
$$

We define a matrix $B$ by

$$
(B \phi)_{\tau}=\frac{1}{\sqrt{2}} \sum_{\theta_{1}=0,1} \phi_{\tau \theta_{1}}
$$


and write

$$
J=\sum_{k=1}^{\Lambda} \frac{\beta_{k}}{\omega^{k}} B^{* k} B^{k}
$$

Precisely saying, $B^{k}$ should be written as a product of distinct matrices $B_{1}, B_{2}, \cdots, B_{k}$. We however suppressed the subscripts of $B$ for simplicity.

\section{A.1 Reflection Positivity}

For $l=1,2, \cdots, \Lambda$, we define the reflection $\rho_{l}$ on the lattice $\mathcal{L}_{\Lambda}$ by

$$
\left(\rho_{l} \theta\right)_{k}=\left\{\begin{array}{ll}
\theta_{k}, & k \neq l, \\
1-\theta_{k}, & k=l,
\end{array} \quad \theta \in \mathcal{L}_{\Lambda} .\right.
$$

Lemma A.1 The measure $\langle\cdot\rangle_{J}$ has reflection positivity with respect to $\rho_{l}, l=1,2, \cdots, \Lambda$.

Remark. Since the reflection planes for $\rho_{l}, l=1,2, \cdots, \Lambda$, separate the $2^{\Lambda}$ points in $\mathcal{L}_{\Lambda}$ from each other, we have "the chessboard bound" 4] used in 9] from this lemma.

Proof. We fix $l$. Let us define the upper half space with respect to $\rho_{l}$ by

$$
\mathcal{L}_{\Lambda}^{+}=\left\{\theta \mid \theta_{l}=1\right\}
$$

and denote the set of all polynomials in $\phi_{\theta}, \theta \in \mathcal{L}_{\Lambda}^{+}$, by $\mathbf{P}^{+}$. The lower half space $\mathcal{L}_{\Lambda}^{-}$and the set $\mathbf{P}^{-}$of all polynomials in $\phi_{\theta}, \theta \in \mathcal{L}_{\Lambda}^{-}$, are similarly defined.

We look into the $k$-th term in the right hand side of A.7. Put

$$
\psi=B^{k} \phi
$$

and write

$$
\left(\phi, B^{* k} B^{k} \phi\right)=\|\psi\|^{2}=\sum_{\theta_{\Lambda}, \ldots, \theta_{k+1}} \psi_{\theta_{\Lambda}, \ldots, \theta_{k+1}}^{2} .
$$

Suppose that $l>k$. Note that

$$
\psi_{\theta_{\Lambda}, \ldots, \theta_{k+1}} \in \begin{cases}\mathbf{P}^{+}, & \theta_{l}=1 \\ \mathbf{P}^{-}, & \theta_{l}=0\end{cases}
$$

Putting

$$
\begin{gathered}
\psi_{+}^{2}=\sum_{\substack{\theta_{\Lambda}, \ldots, \theta_{k+1}=0,1 \\
\theta_{l}=1}} \psi_{\theta_{\Lambda}, \ldots, \theta_{k+1}}^{2}, \\
\psi_{-}^{2}=\sum_{\substack{\theta_{\Lambda}, \ldots, \theta_{k+1}=0,1 \\
\theta_{l}=0}} \psi_{\theta_{\Lambda}, \ldots, \theta_{k+1}}^{2},
\end{gathered}
$$

we have $\psi_{-}^{2}=\rho_{l} \psi_{+}^{2}$ and hence

$$
\|\psi\|^{2}=\psi_{+}^{2}+\psi_{-}^{2}=\psi_{+}^{2}+\rho_{l} \psi_{+}^{2} .
$$

Then, $\left(\phi, B^{* k} B^{k} \phi\right)=\|\psi\|^{2}$ makes a reflection-positive interaction.

Suppose that $l \leq k$. We can decompose $\psi$ as

$$
\psi=\psi^{+}+\psi^{-}, \quad \psi^{ \pm} \in \mathbf{P}^{ \pm},
$$

with

$$
\psi^{-}=\rho_{l} \psi^{+}
$$

Then, we have

$$
\|\psi\|^{2}=\left\|\psi^{+}+\rho_{l} \psi^{+}\right\|^{2}=\left\|\psi^{+}\right\|^{2}+\rho_{l}\left\|\psi^{+}\right\|^{2}+2 \sum_{\theta_{\Lambda}, \ldots, \theta_{k+1}} \psi_{\theta_{\Lambda}, \ldots, \theta_{k+1}}^{+} \rho_{l} \psi_{\theta_{\Lambda}, \ldots, \theta_{k+1}}^{+} .
$$

This means that $\left(\phi, B^{*} B^{k} \phi\right)=\|\psi\|^{2}$ makes a reflection-positive interaction. 


\section{A.2 Hierarchical Laplacian}

We decompose $J$ as

$$
J=\triangle_{\Lambda}+\mu_{0} I
$$

so that

$$
\triangle_{\Lambda} \mathbf{1}_{\Lambda}=0
$$

holds, where $\mu_{0}$ is a constant and $\mathbf{1}_{\Lambda}={ }^{t}(1,1, \cdots, 1) \in \mathbb{R}^{\mathcal{L}_{\Lambda}}$. In fact, using $B \mathbf{1}_{\Lambda-k}=\sqrt{2} \mathbf{1}_{\Lambda-k-1}$, we have

$$
\mu_{0}=\frac{1}{\left\|\mathbf{1}_{\Lambda}\right\|^{2}}\left(\mathbf{1}_{\Lambda}, J \mathbf{1}_{\Lambda}\right)=\sum_{k=1}^{\Lambda} \frac{\beta_{k}}{\omega^{k}}
$$

and hence

$$
-\triangle_{\Lambda}=\sum_{k=1}^{\Lambda} \frac{\beta_{k}}{\omega^{k}}\left(-B^{* k} B^{k}+I\right)=\sum_{k=1}^{\Lambda} \frac{\beta_{k}}{\omega^{k}}\left(-P_{k}+I\right),
$$

where

$$
P_{k}=B^{* k} B^{k}, \quad k=0,1,2, \cdots, \Lambda .
$$

Since $B B^{*}=I$, it holds that

$$
\begin{aligned}
P_{j} P_{k} & =P_{\max (j, k)}, \\
P_{j}^{*} & =P_{j},
\end{aligned}
$$

namely, $P_{j}$ 's are orthogonal projections satisfying

$$
P_{\Lambda}<P_{\Lambda-1}<\cdots<P_{1}<P_{0}=I .
$$

Define $Q_{j}, j=0,1,2, \cdots, \Lambda$, by

$$
\begin{aligned}
Q_{j} & =P_{j}-P_{j+1}, \quad j=0,1,2, \cdots, \Lambda-1 \\
Q_{\Lambda} & =P_{\Lambda}
\end{aligned}
$$

Then, $Q_{0}, Q_{1}, \cdots, Q_{\Lambda}$ constitute orthogonal projections with

$$
\begin{aligned}
Q_{j} Q_{k} & =\delta_{j k} Q_{j}, \\
\sum_{j=0}^{\Lambda} Q_{j} & =I .
\end{aligned}
$$

In this notation, matrices $-\triangle_{\Lambda}+m^{2} I$ and $C=\left(-\triangle_{\Lambda}+m^{2} I\right)^{-1}$ are written as

$$
\begin{aligned}
-\triangle_{\Lambda}+m^{2} I & =\sum_{k=0}^{\Lambda} y_{k} Q_{k}, \quad \text { for } m^{2} \geq 0, \\
C & =\sum_{k=0}^{\Lambda} \frac{1}{y_{k}} Q_{k}, \quad \text { for } m^{2}>0,
\end{aligned}
$$

where

$$
y_{k}= \begin{cases}\sum_{j=k+1}^{\Lambda} \frac{\beta_{j}}{\omega^{j}}+m^{2}, & k=0,1,2, \cdots, \Lambda-1, \\ m^{2}, & k=\Lambda .\end{cases}
$$

Lemma A.2 (1) The matrix $-\triangle_{\Lambda}$ is positive semi-definite and its eigenvalues lie in $\left[0, \mu_{0}\right]$. 
(2) For $l \geq 1$, it holds that

$$
0<\operatorname{Tr} C^{l} \leq \frac{2^{\Lambda}}{m^{2 l}} .
$$

Proof. (1) Put $m^{2}=0$ in (A.12) and (A.14). Then, we see that the smallest eigenvalue of $-\triangle_{\Lambda}$ is 0 and the largest one is

$$
\sum_{j=1}^{\Lambda} \frac{\beta_{j}}{\omega^{j}}=\mu_{0}
$$

(2) Note that A.13 implies

$$
\operatorname{Tr} C^{l}=\sum_{k=0}^{\Lambda} \frac{1}{y_{k}^{l}} \operatorname{Tr} Q_{k}=\sum_{k=0}^{\Lambda-1} \frac{2^{\Lambda-k-1}}{y_{k}^{l}}+\frac{1}{y_{\Lambda}^{l}}, \quad l=1,2, \cdots .
$$

Since A.14 implies $y_{k} \geq m^{2}$, we can estimate the right hand side of A.15.

Based on Lemma A.1 and Lemma A.2 we can apply the $1 / N$ expansion 9 to the hierarchical system and obtain Proposition 5.3

Proof of Lemma 5.6. (1) Note that A.13) and the equality

$$
B^{\Lambda} Q_{j} B^{* \Lambda}= \begin{cases}0, & 0 \leq j \leq \Lambda-1 \\ 1, & j=\Lambda\end{cases}
$$

yield

$$
B^{\Lambda} C B^{* \Lambda}=\frac{1}{m^{2}} .
$$

This means 5.30).

(2) Since

$$
\left(Q_{j}\right)_{\theta \theta}=\left\{\begin{array}{ll}
2^{-j-1}, & 0 \leq j \leq \Lambda-1, \\
2^{-\Lambda}, & j=\Lambda,
\end{array} \quad \theta \in \mathcal{L}_{\Lambda},\right.
$$

A.13) implies

$$
C_{\theta \theta}=\sum_{k=0}^{\Lambda-1} \frac{1}{2^{k+1} y_{k}}+\frac{1}{2^{\Lambda} y_{\Lambda}}, \quad \theta \in \mathcal{L}_{\Lambda} .
$$

Now, under A.5 we have

$$
y_{k}= \begin{cases}\frac{1}{2 \omega^{\Lambda}}\left(\omega^{\Lambda-k}-\omega+4 t+2 \omega^{\Lambda} m^{2}\right), & 0 \leq k \leq \Lambda-1, \\ m^{2}, & k=\Lambda .\end{cases}
$$

Then, it holds that

$$
C_{\theta \theta}=\left(\frac{\omega}{2}\right)^{\Lambda} \sum_{j=1}^{\Lambda} \frac{2^{j}}{\omega^{j}-\omega+4 t+2 \omega^{\Lambda} m^{2}}+\frac{1}{2^{\Lambda} m^{2}} .
$$




\section{References}

[1] P. M. Bleher, Ya. G. Sinai, Investigation of the critical point in models of the type of Dyson's hierarchical model, Commun. Math. Phys. 33 (1973) 23-42.

[2] P. M. Bleher, Ya. G. Sinai, Critical indices for Dyson's asymptotically hierarchical models, Commun. Math. Phys. 45 (1975) 247-278.

[3] F. J. Dyson, Existence of a phase-transition in a one-dimensional Ising ferromagnet, Commun. Math. Phys. 12 (1969) 91-107.

[4] J. Fröhlich, R. Israel, E. H. Lieb, B. Simon, Phase transitions and reflection positivity. I. General theory and long range lattice models, Commun. Math. Phys. , 62, 1978, 1-34.

[5] K. Gawędzki, A. Kupiainen, Non-Gaussian fixed points of the block spin transformation. Hierarchical model approximation, Commun. Math. Phys. 89 (1983) 191-220.

[6] K. Gawędzki, A. Kupiainen, Continuum Limit of the Hierarchical $O(N)$ Non-Linear $\sigma$-Model, Commun. Math. Phys. 106 (1986) 533-550.

[7] T. Hara, T. Hattori, H. Watanabe, Triviality of Hierarchical Ising Model in Four Dimensions, Commun. Math. Phys. 220 (2001) 13-40.

[8] Y. V. Kozitsky, Hierarchical model of a vector ferromagnet. Self-similar block-spin distributions and the Lee-Yang theorem, Reports on Mathematical Physics, 26 (1988) 429-445.

[9] A. J. Kupiainen, On the 1/n expansion, Commun. Math. Phys. , 73, 1980, 273-294.

[10] C. M. Newman Inequalities for Ising models and field theories which obey the Lee-Yang theorem, Commun. Math. Phys. 41 (1975) 1-9.

[11] Ya. G. Sinai, Theory of phase transition: rigorous results, Pergamon Press, 1982. 